\title{
A Moving Horizon Approach to Networked Control System Design
}

\author{
Graham C. Goodwin, Fellow, IEEE, Hernan Haimovich, Student Member, IEEE, \\ Daniel E. Quevedo, Student Member, IEEE, and James S. Welsh, Member, IEEE
}

\begin{abstract}
This paper presents a control system design strategy for multivariable plants where the controller, sensors and actuators are connected via a digital, data-rate limited, communications channel. In order to minimize bandwidth utilization, a communication constraint is imposed which restricts all transmitted data to belong to a finite set and only permits one plant to be addressed at a time. We emphasize implementation issues and employ moving horizon techniques to deal with both control and measurement quantization issues. We illustrate the methodology by simulations and a laboratory-based pilot-scale study.
\end{abstract}

Index Terms-Centralized control, control over networks, digital control, limited communications, moving horizon estimation, networked control systems, optimal control, predictive control, quantization, set-valued observers, stability, vector quantization.

\section{INTRODUCTION}

$\mathbf{N}$ ETWORKED control systems (NCSs) are control systems in which controller and plant are connected via a communication channel; see, e.g., [1] and [2]. While the use of digital communication channels enables novel teleoperating applications, new and interesting challenges also arise. The network itself is a dynamical system that exhibits characteristics which traditionally have not been taken into account in control system design. These special characteristics include quantization and time-delays and are a consequence of the fact that practical channels have limited bandwidth. A successful NCS design should take network characteristics into account.

Due to the digital nature of the communication channel, every transmitted signal is quantized to a finite set; see, e.g., [2]. In some situations the quantization effect may be neglected or approximated by white noise [3], [4]. However, this is not appropriate when only a few quantization levels are available; see, e.g., [5]-[9]. Hence, we argue that the finite set nature of the data should be explicitly taken into account in the design of NCS.

Another consequence of utilizing channels with limited bandwidth is the introduction of time-delays. Since the medium is data-rate limited, signals may have to queue before being transmitted, leading to delays in the up-link (from sensors to controller) and in the down-link (from controller to actuators). Without considering quantization effects, several problems have been studied in the literature; see, e.g., [10] and [11].

Manuscript received May 3, 2003; revised December 14, 2003 and March 15, 2004. Recommended by Guest Editors P. Antsaklis and J. Baillieul.

The authors are with the School of Electrical Engineering and Computer Science, The University of Newcastle, Callaghan, NSW 2308, Australia (e-mail: eegcg@ee.newcastle.edu.au; hhaimo@ee.newcastle.edu.au; dquevedo@ieee.org; jwelsh@ee.newcastle.edu.au).

Digital Object Identifier 10.1109/TAC.2004.834132
In many cases, it is possible to time-stamp the up-link data [12], so that the delays are known at the receiving end. Thus, (without taking into account quantization issues) plant state estimates can be obtained, at the controller side, by simply solving a standard Kalman filtering problem [13]. On the other hand, time-stamping is not usually helpful in order to compensate for delays in the down-link, since they occur after the control calculations. Thus, deterministic down-link traffic is more important for performance. In order to obtain fixed down-link delays, up-link data may be given lower priority and overall traffic should be kept at a minimum. This can be achieved by deploying event-based (nonuniform) sampling of the plant outputs, as described in [14]. Furthermore, the use of quantization limits the data rate requirements and, hence, reduces network traffic.

The specific problem addressed here is the design of a methodology for minimizing network traffic between a centralized controller and a multivariable plant, or collection of plants. We split the design problem into two subproblems, namely, up-link and down-link. In both cases, we utilize Moving Horizon techniques in order to deal with the associated computational issues; see, e.g., [15]-[19].

On the up-link side, we employ signal quantization to minimize data rate requirements. In particular, at each sampling time, we transmit data specifying only a region in which the measurements lie. An extreme case would be the transmission of only one bit per sensor indicating that the measurement lies either above or below some prespecified threshold value (e.g., set point). We propose to address the nonlinear filtering problem by using one of two estimation schemes. One method utilizes concepts from Monte Carlo (MC) sampling [20] and aims to obtain the conditional expectation of the current state given the quantized output history. The other method is deterministic and utilizes an unknown-but-bounded description of uncertainty (see [21]-[23]). For this case, we design a recursive set-valued observer which is aimed at finding the smallest region of state space in which the state is guaranteed to lie. Our approach is closely related to bounded-error estimation (see, e.g., [24] and [25]) but our development is directly tailored to systems having quantized measurements. In both methods, we utilize a moving horizon concept. We explicitly consider only a fixed number of the most recent measurements and summarize previous data via a suitable description for a previous state. In the Monte Carlo method, this description takes the form of a Gaussian distribution, whereas in the deterministic method it takes the form of a bounding region. The choice between 
these two alternatives in practice is a function of the nature of the available prior data regarding the noise model (stochastic or deterministic) and computational issues (see discussion in Sections IV and V).

On the down-link side, we restrict the controller such that only one actuator can be addressed at any given time (compare to limited communication control [26], [27]). Moreover, only one of a finite number of levels can be transmitted. The design of the resulting system is aimed at optimizing performance subject to these constraints. In this context, we choose to send increments in the control signal, rather than the actual values. Between updates, all inputs are kept at their previous values. An extreme case would be that we transmit only one bit per actuator indicating that the plant input should be increased or decreased. Our strategy is characterized by the fact that we do not fix the control law a priori, but instead determine the plant inputs via a moving horizon online optimization, which takes account of, inter-alia, disturbances, speed of response, open loop unstable poles, etc. A key, and distinguishing feature is that we use a finite-set constraint for all control moves, thus limiting the number of bits needed to be transmitted at any one time. This set constraint puts the problem into the framework of, so called, finite alphabet control problems; see, e.g., [28] and the references therein.

Our scheme is particularly suited to protocols where the message size can be manipulated. However, this does not mean that bandwidth reduction via our proposal is only a consequence of minimizing message length. Bandwidth is also conserved due to the dynamic optimization of the system with respect to supplying control increments only when they are required.

The main contribution of this work resides in applying moving horizon concepts to quantized NCS in a practical context. Moving horizon ideas allow one to trade off computational complexity with performance. This paper combines and extends ideas from three recent conference papers by the authors; see [29]-[31].

The layout of this paper is as follows. In Section II, the design issues of NCS associated with quantized measurements in the up-link are addressed. We describe a stochastic estimator structure in Section II-A and its deterministic counterpart in Section II-B. Section III describes our proposed down-link control strategy which relies on solving a finite set constrained optimization problem. In Section IV, we analyze the proposed control strategy via computer simulations. Section V documents a laboratory-scale experiment which illustrates the main characteristics of the proposed strategy. Conclusions are drawn in Section VI.

\section{UP-LINK DESIGN}

In this section, we propose two alternative design strategies for dealing with quantized measurements in the up-link. First, we adopt a stochastic problem formulation and develop an approximate minimum variance state estimator. Second, by adopting a deterministic formulation, we propose a set-valued observer and select a Chebyshev center of the computed set as a point state estimate.
In the sequel, let $k$ denote the current sample time and $\overleftarrow{\mathbf{y}}_{a: b}$ past values of the variable $y$ from sample time $a$ to $b$ inclusive. Furthermore, we use $\mathbf{N}(\mu, P)$ to denote a multivariate Gaussian probability density function (pdf) with mean vector $\mu$ and covariance matrix $P$, and $\mathbf{E}()$ to denote the expectation operator. Also, $I_{n}$ denotes the identity matrix in $\mathbb{R}^{n \times n}$.

\section{A. Stochastic Formulation}

1) Problem Statement: We consider a noisy discrete-time linear time-invariant plant described by

$$
\begin{aligned}
x_{j+1} & =A x_{j}+B u_{j}+w_{j} \\
y_{j} & =C x_{j}+v_{j}
\end{aligned}
$$

where $x_{j}, w_{j} \in \mathbb{R}^{n}, u_{j} \in \mathbb{R}^{m}, y_{j}, v_{j} \in \mathbb{R}^{s}$, and $w_{j}$ and $v_{j}$ are i.i.d. samples of white Gaussian noise processes having covariance matrices $Q$ and $R$, respectively. We further assume that the measurements are quantized prior to transmission

$$
y_{j}^{q}=\sigma\left(y_{j}\right)
$$

where $y_{j}^{q}$ is the measured variable. Here, the finite-range mapping $\sigma(\cdot)$ corresponds to a general quantizer, defined as

$$
\sigma\left(y_{j}\right)=\left\{\begin{array}{cccc}
\sigma_{1}, & \text { if } & y_{j} \in \mathcal{R}_{1} \\
\sigma_{2}, & \text { if } & y_{j} \in \mathcal{R}_{2} \\
\vdots & \vdots & \vdots \\
\sigma_{\mathrm{L}}, & \text { if } & y_{j} \in \mathcal{R}_{\mathrm{L}}
\end{array}\right.
$$

where $\mathrm{L}$ is the number of quantization regions and $\left\{\mathcal{R}_{i}: i=\right.$ $1, \ldots, \mathrm{L}\}$ is a partition of $\mathbb{R}^{s}$, which we assume to be fixed and known to the controller.

The statistical behavior of the system is described by

$$
x_{o} \sim \mathbf{N}\left(x_{o o}, P_{o o}\right) \quad w_{i} \sim \mathbf{N}(0, Q) \quad v_{i} \sim \mathbf{N}(0, R)
$$

and by $\mathbf{E}\left(w_{i} w_{j}^{T}\right)=0$ and $\mathbf{E}\left(v_{i} v_{j}^{T}\right)=0$, if $i \neq j$, and $\mathbf{E}\left(w_{i} v_{j}^{T}\right)=0, \mathbf{E}\left(w_{i} x_{o}^{T}\right)=0, \mathbf{E}\left(v_{i} x_{o}^{T}\right)=0$, if $i, j=0,1, \ldots$.

Remark 1: Note that we have assumed that (quantized) measurements are available at each discrete time-step. However, it would be a straightforward extension to also use time-stamped data with event-based sampling to further limit transmission rates so that we only transmit when a measurement changes zones.

2) Classical State Estimation With Output Quantization: We focus on determining the conditional mean of the current state, $x_{k}$, given the collection of measurements from time instant 1 to the present time instant, $k$. The required state estimate at time instant $k$ is given by

$$
\begin{aligned}
\hat{x}_{k} & \triangleq \mathbf{E}\left[x_{k} \mid \overleftarrow{\mathbf{y}}_{1: k}^{q}\right] \\
& =\mathbf{E}\left[x_{k} \mid y_{1}^{q}, \ldots, y_{k}^{q}\right] \\
& =\mathbf{E}\left[x_{k} \mid y_{1} \in \sigma^{-1}\left(y_{1}^{q}\right), \ldots, y_{k} \in \sigma^{-1}\left(y_{k}^{q}\right)\right]
\end{aligned}
$$

where $\sigma^{-1}\left(y^{q}\right)$ denotes the quantization region corresponding to the measurement $y^{q}$, i.e $\sigma^{-1}\left(\sigma_{i}\right)=\mathcal{R}_{i}$, for $i=1, \ldots, \mathrm{L}$ [see (3)]. 
As reported in [4], the estimate given by (5) can be obtained in a conceptually simple way by taking advantage of the linear structure which exists between system input, state and unavailable (unmeasured) variable $y_{k}$. Indeed, using the concatenation property of the expectation operator, we have

$$
\mathbf{E}\left[x_{k} \mid \overleftarrow{\mathbf{y}}_{1: k}^{q}\right]=\mathbf{E}\left[\mathbf{E}\left(x_{k} \mid \overleftarrow{\mathbf{y}}_{1: k}\right) \mid \overleftarrow{\mathbf{y}}_{1: k}^{q}\right]
$$

The inner expected value on the right-hand side of (6) is provided by the equations of the Kalman filter [32], [33], which are summarized in (7)-(13).

$$
\begin{aligned}
& \mathbf{E}\left(x_{o}\right) \triangleq x_{o o} \\
& P_{o \mid o} \triangleq \\
& P_{o o} \\
& \mathbf{E}\left(x_{k} \mid \overleftarrow{\mathbf{y}}_{1: k-1}\right)=A \mathbf{E}\left(x_{k-1} \mid \overleftarrow{\mathbf{y}}_{1: k-1}\right)+B u_{k-1} \\
& P_{k \mid k-1}= A P_{k-1 \mid k-1} A^{T}+Q \\
& K_{k}= P_{k \mid k-1} C^{T}\left(C P_{k \mid k-1} C^{T}+R\right)^{-1} \\
& \mathbf{E}\left(x_{k} \mid \overleftarrow{\mathbf{y}}_{1: k}\right)= \mathbf{E}\left(x_{k} \mid \overleftarrow{\mathbf{y}}_{1: k-1}\right) \\
&+K_{k}\left[y_{k}-C \mathbf{E}\left(x_{k} \mid \overleftarrow{\mathbf{y}}_{1: k-1}\right)\right] \\
& P_{k \mid k}=\left(I_{n}-K_{k} C\right) P_{k \mid k-1} \cdot
\end{aligned}
$$

The analysis that follows depends on interpreting $\mathbf{E}\left(x_{k} \mid \overleftarrow{\mathbf{y}}_{1: k}\right)$ as a function of the random variable $\overleftarrow{\mathbf{y}}_{1: k}$ rather than as a function of a given realization of the output, $\left\{y_{j}\right\}$. Once this function is determined, the outer expected value in (6) has to be evaluated as shown in (14) at the bottom of the page, where $\rho_{1: k}$ is the region $\sigma^{-1}\left(y_{1}^{q}\right) \times \cdots \times \sigma^{-1}\left(y_{k}^{q}\right)$ (contained in $\mathbb{R}^{s \times k}$ ), and $p_{y_{1}, \ldots, y_{k}}\left(\gamma_{1}, \ldots, \gamma_{k}\right)$ is the joint pdf of the random variables $y_{1}, \ldots, y_{k}$. This joint pdf can be found as described in Lemma 1. Indeed, we make a slight generalization for future use, where we assume $x_{k-l} \sim \mathbf{N}\left(x_{k-l}^{\prime}, P_{k-l}^{\prime}\right)$. From (7) and (8), we clearly see that this holds when $l=k$, with $x_{k-l}^{\prime}=x_{o o}$ and $P_{k-l}^{\prime}=P_{o o}$.

Lemma 1: Let $k \geq l \geq 1$ and assume $x_{k-l} \sim$ $\mathbf{N}\left(x_{k-l}^{\prime}, P_{k-l}^{\prime}\right)$. Then, the joint pdf of the random variables $y_{k-l+1}, \ldots, y_{k}$ is Gaussian, having mean vector

$$
\mathbf{E}\left(\overleftarrow{\mathbf{y}}_{k-l+1: k}\right)=\Lambda_{l} x_{k-l}^{\prime}+\Phi_{l} \overleftarrow{\mathbf{u}}_{k-l: k-1}
$$

and covariance matrix shown in (16) at the bottom of the page, where

$$
\begin{aligned}
& \operatorname{cov}\left(y_{k-l+i}, y_{k-l+j}\right)=C A^{i} P_{k-l}^{\prime}\left(C A^{j}\right)^{T} \\
& +\sum_{l=1}^{\min \{i, j\}} C A^{i-l} Q\left(C A^{j-l}\right)^{T}+\delta_{i j} R, \quad i, j=1, \ldots, l
\end{aligned}
$$

with $\delta_{i j}$ the Kronecker delta and where $\Lambda_{l}$ and $\Phi_{l}$ are suitable constant matrices whose dimensions are $l s \times n$ and $l s \times l m$, respectively.

Proof: This is a straightforward consequence of the linearity of the system and properties of Gaussian variables under linear operations; see also [29].

As a consequence of this result (when $l=k$ ), the desired estimate could, in principle, be obtained by performing the multivariate integration shown in (14). However, direct application of (14) becomes impractical for the reasons expressed in the following two remarks.

Remark 2: The multiple integration in (14) has to be solved numerically since, in general, no explicit closed-form solution exists.

Remark 3: As new measurements become available, the complexity of the integration in (14) increases since it is performed over the region $\rho_{1: k}$, contained in $\mathbb{R}^{s \times k}$, where $k$ denotes the current time.

In the sequel, we bring together ideas from MC sampling [20] and moving horizon estimation [18], [34]. These ideas are utilized in order to address the problems described in Remarks 2 and 3 .

3) Moving Horizon Monte Carlo State Estimation: Remarks 2 and 3 highlight the problems encountered when the estimate $\mathbf{E}\left[x_{k} \mid \overleftarrow{\mathbf{y}}_{1: k}^{q}\right]$ needs to be calculated. In this section, we discuss a practical approach to finding state estimates. The issue of solving the multivariate integrals in (14) is addressed in Section II-A3a while the growing complexity problem is dealt with in Section II-A3b.

a) Monte Carlo sampling: To provide a numerical solution to (14), we propose the use of Monte Carlo sampling. Let $\mathrm{N}_{\mathrm{S}}$ be a positive integer and denote a set of $\mathrm{N}_{\mathrm{S}}$ samples drawn from the Gaussian pdf $p_{y_{1}, \ldots, y_{k}}\left(\gamma_{1}, \ldots, \gamma_{k}\right)$ at time instant $k$ by

$$
\begin{aligned}
\mathbf{E}\left[x_{k} \mid \overleftarrow{\mathbf{y}}_{1: k}^{q}\right] & =\mathbf{E}\left[\mathbf{E}\left(x_{k} \mid \overleftarrow{\mathbf{y}}_{1: k}\right) \mid \overleftarrow{\mathbf{y}}_{1: k}^{q}\right] \\
& =\frac{\int_{\rho_{1: k}} p_{y_{1}, \ldots, y_{k}}\left(\gamma_{1}, \ldots, \gamma_{k}\right) \mathbf{E}\left[x_{k} \mid \overleftarrow{\mathbf{y}}_{1: k}=\left(\gamma_{1}, \ldots, \gamma_{k}\right)\right] d \gamma_{1} \ldots d \gamma_{k}}{\int_{\rho_{1: k}} p_{y_{1}, \ldots, y_{k}}\left(\gamma_{1}, \ldots, \gamma_{k}\right) d \gamma_{1} \ldots d \gamma_{k}}
\end{aligned}
$$

$$
\operatorname{cov}\left(\overleftarrow{\mathbf{y}}_{k-l+1: k}\right)=\left[\begin{array}{cccc}
\operatorname{cov}\left(y_{k-l+1}, y_{k-l+1}\right) & \operatorname{cov}\left(y_{k-l+1}, y_{k-l+2}\right) & \cdots & \operatorname{cov}\left(y_{k-l+1}, y_{k}\right) \\
\operatorname{cov}\left(y_{k-l+2}, y_{k-l+1}\right) & \operatorname{cov}\left(y_{k-l+2}, y_{k-l+2}\right) & \cdots & \operatorname{cov}\left(y_{k-l+2}, y_{k}\right) \\
\vdots & \vdots & \ddots & \vdots \\
\operatorname{cov}\left(y_{k}, y_{k-l+1}\right) & \operatorname{cov}\left(y_{k}, y_{k-l+2}\right) & \cdots & \operatorname{cov}\left(y_{k}, y_{k}\right)
\end{array}\right]
$$


$S_{k}$. Define $S_{\rho_{1: k}} \subset S_{k}$ as the set of samples that fall inside the region $\rho_{1: k}$, i.e.,

$$
S_{\rho_{1: k}} \triangleq S_{k} \cap \rho_{1: k}
$$

Equation (14) can then be approximated by

$$
\mathbf{E}\left[x_{k} \mid \overleftarrow{\mathbf{y}}_{1: k}^{q}\right] \approx \frac{1}{\mathrm{~N}_{\rho_{1: k}}} \sum_{\overleftarrow{\mathbf{z}} \in S_{\rho_{1: k}}} \mathbf{E}\left(x_{k} \mid \overleftarrow{\mathbf{y}}_{1: k}=\overleftarrow{\mathbf{z}}\right)
$$

where $\mathrm{N}_{\rho_{1: k}}$ is the number of samples that fall inside the region $\rho_{1: k}$, i.e., the cardinality of the set $S_{\rho_{1: k}}$. The closeness of the Monte Carlo approximation to the estimate (5) will directly depend on $\mathrm{N}_{\rho_{1: k}}$ : larger values of this number yield better approximations. See [29] for an informative example.

b) Moving horizon approach: As far as growing complexity is concerned (Remark 3), we adopt a moving horizon approach. At time instant $k$, we explicitly take into account only the last $\mathrm{N}_{\mathrm{M}}$ measurements available, $\overleftarrow{\mathbf{y}}_{k-\mathrm{N}_{\mathrm{M}}+1: k}^{q}$, whereas the older measurements, $\overleftarrow{\mathbf{y}}_{1: k-\mathrm{N}_{\mathrm{M}}}^{q}$, will be dealt with by summarizing the history of the system before time instant $k-\mathrm{N}_{\mathrm{M}}$. This summarizing step is performed by adopting a suitable conditional distribution for the state $x_{k-\mathrm{N}_{\mathrm{M}}}$. Consequently, at time instant $k$, the state $x_{k-\mathrm{N}_{\mathrm{M}}}$ will be regarded as the initial state. The parameter $\mathrm{N}_{M}$ will be called the measurement horizon and will allow us to trade off observer performance for computational complexity.

In (4), the Gaussian distribution ascribed to the initial state, $x_{o}$, can be interpreted as the summary of the system history prior to time instant 1 . This Gaussianity assumption for the initial state, together with the corresponding ones for the process and measurement noises and the linearity of the system defined in (1) rendered the equations of the Kalman filter suitable for determining the expected value $\mathbf{E}\left(x_{k} \mid \overleftarrow{\mathbf{y}}_{1: k}\right)$, needed for the two-step evaluation procedure described in (6).

It follows from the Chapman-Kolmogorov equations (time and observation update equations, see, e.g., [35]) that knowledge of the conditional density of the state $x_{k-\mathrm{N}_{\mathrm{M}}}$, of the form $p\left(x_{k-\mathrm{N}_{\mathrm{M}}} \mid \overleftarrow{\mathbf{y}}_{1: k-\mathrm{N}_{\mathrm{M}}}^{q}\right)$, is all that is needed in order to summarize the history of the system if we are interested only in subsequent estimates of the form

$$
\mathbf{E}\left[x_{k-\mathrm{N}_{\mathrm{M}}+i} \mid \overleftarrow{\mathbf{y}}_{1: k-\mathrm{N}_{\mathrm{M}}+i}^{q}\right], \quad i=1,2, \ldots
$$

Due to the quantized nature of the measurements, the conditional density of the state $x_{k-\mathrm{N}_{\mathrm{M}}}$ given the measurements $\overleftarrow{\mathbf{y}}_{1: k-\mathrm{N}_{\mathrm{M}}}^{q}$ is not Gaussian. However, approximating this density as Gaussian enables us to utilize the two-step procedure shown in (6) because the inner expected value can again be provided by the equations of the Kalman filter, regarding $x_{k-\mathrm{N}_{\mathrm{M}}}$ as the initial state.

c) Proposed stochastic estimator: The proposed approach brings together the ideas presented in Sections II-A3a and II-A3b. The initial estimates $\mathbf{E}\left[x_{k} \mid \overleftarrow{\mathbf{y}}_{1: k}^{q}\right]$ for $k=1, \ldots, \mathrm{N}_{\mathrm{M}}$ are found exactly as described in the preceding sections and the multivariate integrals in (14) are approximated with the aid of MC sampling. From time instant $\mathrm{N}_{\mathrm{M}}+1$ on, the conditional densities $p\left(x_{k-\mathrm{N}_{\mathrm{M}}} \mid \overleftarrow{\mathbf{y}}_{1: k-\mathrm{N}_{\mathrm{M}}}^{q}\right)$ are approximated by the Gaussian pdf $\mathbf{N}\left(x_{k-\mathrm{N}_{\mathrm{M}}}^{\prime}, P_{k-\mathrm{N}_{\mathrm{M}}}^{\prime}\right)$. Equations (7) and (8) are replaced by

$$
\begin{array}{r}
\mathbf{E}\left(x_{k-\mathrm{N}_{\mathrm{M}}} \mid \overleftarrow{\mathbf{y}}_{1: k-\mathrm{N}_{\mathrm{M}}}\right) \triangleq x_{k-\mathrm{N}_{\mathrm{M}}}^{\prime} \\
P_{k-\mathrm{N}_{\mathrm{M}} \mid k-\mathrm{N}_{\mathrm{M}}} \triangleq P_{k-\mathrm{N}_{\mathrm{M}}}^{\prime}
\end{array}
$$

while (9) to (13) remain unchanged. The approximate estimate of $\mathbf{E}\left[x_{k} \mid \overleftarrow{\mathbf{y}}_{1: k}^{q}\right]$ is then obtained by performing the multivariate integrals, as shown in (23) at the bottom of the page, with the aid of Monte Carlo sampling, where $\rho_{k-\mathrm{N}_{\mathrm{M}}+1: k}$ is the region $\sigma^{-1}\left(y_{k-\mathrm{N}_{\mathrm{M}}+1}^{q}\right) \times \cdots \times \sigma^{-1}\left(y_{k}^{q}\right)$ (contained in $\left.\mathbb{R}^{s \times \mathrm{N}_{\mathrm{M}}}\right)$ and $f\left(\gamma_{1}, \ldots, \gamma_{\mathrm{N}_{\mathrm{M}}}\right) \triangleq p_{y_{k-\mathrm{N}_{\mathrm{M}}+1}, \ldots, y_{k}}\left(\gamma_{1}, \ldots, \gamma_{\mathrm{N}_{\mathrm{M}}}\right)$ is now the joint pdf of the variables $y_{k-\mathrm{N}_{\mathrm{M}}+1}, \ldots, y_{k}$ under the Gaussianity assumption for $x_{k-\mathrm{N}_{\mathrm{M}}}$. This density has been quantified in Lemma 1 (setting $l=\mathrm{N}_{\mathrm{M}}$ ).

The suggested strategy can be regarded as a generalization of the Gaussian fit algorithm described in [4]. A graphical representation of the method proposed is included in Fig. 1.

The values adopted for the parameters $x_{k-\mathrm{N}_{\mathrm{M}}}^{\prime}$ and $P_{k-\mathrm{N}_{\mathrm{M}}}^{\prime}$ will affect the estimate sought. We propose to adopt the quantity $x_{k-\mathrm{N}_{\mathrm{M}}}^{\prime}$ as the estimate of $x_{k-\mathrm{N}_{\mathrm{M}}}$ found $\mathrm{N}_{\mathrm{M}}$ time instants before. As regards $P_{k-\mathrm{N}_{\mathrm{M}}}^{\prime}$, different options can be considered.

1) $P_{k-\mathrm{N}_{\mathrm{M}}}^{\prime}=0$. In this case, perfect knowledge of $x_{k-\mathrm{N}_{\mathrm{M}}}$ is assumed.

2) $P_{k-\mathrm{N}_{\mathrm{M}}}^{\prime} \rightarrow \infty$. This corresponds to assuming very little precision of the estimate of the initial state.

3) $0<P_{k-\mathrm{N}_{\mathrm{M}}}^{\prime}<\infty$, arbitrary. Here, $P_{k-\mathrm{N}_{\mathrm{M}}}^{\prime}$ is regarded as a tuning parameter.

4) $P_{k-\mathrm{N}_{\mathrm{M}}}^{\prime} \approx \operatorname{cov}\left(x_{k-\mathrm{N}_{\mathrm{M}}} \mid \overleftarrow{\mathbf{y}}_{1: k-\mathrm{N}_{\mathrm{M}}}^{q}\right)$. Here, $P_{k-\mathrm{N}_{\mathrm{M}}}^{\prime}$ is set equal to an approximation of the true conditional covariance of the initial state.

Note that option 1) is analogous to the idea of decision feedback equalizers in digital communications; see, e.g., [36]. Also note that option 4) need not give better results than a different choice for $P_{k-\mathrm{N}_{\mathrm{M}}}^{\prime}$ because the true conditional distribution of the state $x_{k-\mathrm{N}_{\mathrm{M}}}$ given the measurements $\overleftarrow{\mathbf{y}}_{1: k}^{q}$ is not Gaussian.

The procedure previously described can be summarized in the following algorithm, which is to be performed when $k>\mathrm{N}_{\mathrm{M}}$.

$$
\mathbf{E}\left[x_{k} \mid \overleftarrow{\mathbf{y}}_{k}^{q}\right] \approx \frac{\int_{\rho_{k-\mathrm{N}_{\mathrm{M}}+1: k}} f\left(\gamma_{1}, \ldots, \gamma_{\mathrm{N}_{\mathrm{M}}}\right) \mathbf{E}\left[x_{k} \mid \overleftarrow{\mathbf{y}}_{k-\mathrm{N}_{\mathrm{M}}+1: k}=\left(\gamma_{1}, \ldots, \gamma_{\mathrm{N}_{\mathrm{M}}}\right)\right] d \gamma_{1} \ldots d \gamma_{\mathrm{N}_{\mathrm{M}}}}{\int_{\rho_{k-\mathrm{N}_{\mathrm{M}}+1: k}} f\left(\gamma_{1}, \ldots, \gamma_{\mathrm{N}_{\mathrm{M}}}\right) d \gamma_{1} \ldots d \gamma_{\mathrm{N}_{\mathrm{M}}}}
$$




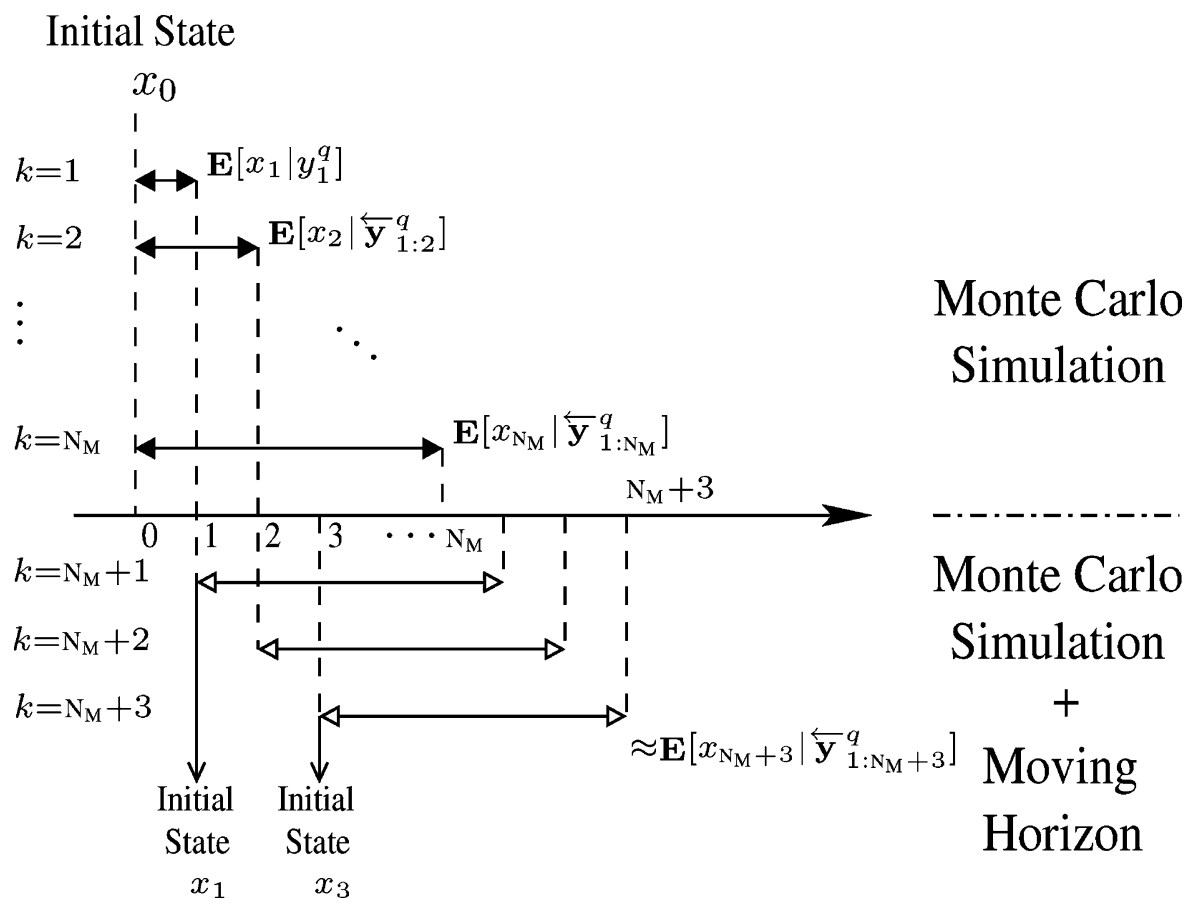

Fig. 1. Moving horizon MC state estimation strategy.

Algorithm 1 (Moving Horizon MC state estimation)

1) Adopt the parameters for the initial state distribution $\left(x_{k-\mathrm{N}_{\mathrm{M}}}^{\prime}\right.$ and $P_{k-\mathrm{N}_{\mathrm{M}}}^{\prime}$ ).

2) Calculate the mean and covariance of $\overleftarrow{\mathbf{y}}_{k-\mathrm{N}_{\mathrm{M}}+1: k}$ as in (15) and (16).

3) Draw $N_{S}$ samples from

$$
\mathbf{N}\left(\mathbf{E}\left[\overleftarrow{\mathbf{y}}_{k-\mathrm{N}_{\mathrm{M}}+1: k}\right], \mathbf{c o v}\left[\overleftarrow{\mathbf{y}}_{k-\mathrm{N}_{\mathrm{M}}+1: k}\right]\right)
$$

and determine which ones fall inside

the region $\rho_{k-\mathrm{N}_{\mathrm{M}}+1: k}$.

4) Approximate $\mathbf{E}\left[x_{k} \mid \overleftarrow{\mathbf{y}}_{1: k}^{q}\right]$ as in (23) and (19).

5) Set $k \leftarrow k+1$ and go to step 1 .

Remark 4: In a NCS architecture, we cannot expect to be able to utilize measured signals up to the current time instant, $k$, in order to calculate the control action $u_{k}$. Indeed, due to communication constraints and computation time we should, at least, allow for a unit time delay. To that extent, it is useful to formulate a one-step-ahead state estimate, namely $\mathbf{E}\left[x_{k+1} \mid \overleftarrow{\mathbf{y}}_{1: k}^{q}\right]$. This estimate can easily be obtained by using (1), the linearity of the expectation operator and the fact that $\mathbf{E}\left[w_{k} \mid \overleftarrow{\mathbf{y}}_{1: k}^{q}\right]=0$, via

$$
\mathbf{E}\left[x_{k+1} \mid \overleftarrow{\mathbf{y}}_{1: k}^{q}\right]=A \mathbf{E}\left[x_{k} \mid \overleftarrow{\mathbf{y}}_{1: k}^{q}\right]+B u_{k}
$$

Remark 5: It is important to note how the computational complexity of the proposed method depends on the measurement horizon, $\mathrm{N}_{\mathrm{M}}$. By increasing this parameter, the dimension of the space over which the integral (23) is performed increases. To approximate this integral by means of MC sampling, we need to draw samples from a Gaussian distribution in this space, but only the samples belonging to the region $\rho_{k-\mathrm{N}_{\mathrm{M}}+1: k}$ will be considered. To obtain a sensible state estimate, the number of samples to be drawn from this Gaussian distribution has to be sufficiently large to allow for a substantial number of samples to fall inside the region $\rho_{k-\mathrm{N}_{\mathrm{M}}+1: k}$. To give an idea of the magnitude of this problem, consider a quantizer with only two quantization regions $[\mathrm{L}=2$, see (3)] and a measurement horizon $\mathrm{N}_{\mathrm{M}}=10$. This yields $\mathrm{L}^{\mathrm{N}_{\mathrm{M}}}=1024$ different regions in $\mathbb{R}^{s \times \mathrm{N}_{\mathrm{M}}}$, according to the possible values of the quantized measurements throughout the measurement horizon.

\section{B. Deterministic Formulation}

1) Problem Statement: Here, we consider an alternative deterministic discrete-time linear time-invariant system with quantized state measurements

$$
\begin{aligned}
x_{j+1} & =A x_{j}+B u_{j}+w_{j} \\
y_{j}^{q} & =\sigma\left(x_{j}+v_{j}\right)
\end{aligned}
$$

where, as before, $x_{j} \in \mathbb{R}^{n}, u_{j} \in \mathbb{R}^{m}$, and $y_{j}^{q}$ is the measured variable. $\left\{v_{j}\right\}$ and $\left\{w_{j}\right\}$ are unknown sequences whose elements comply with

$$
\begin{aligned}
\left\|w_{j}\right\|_{t} \leq \delta, & j=0,1, \ldots \\
\left\|v_{j}\right\|_{t} \leq \epsilon, & j=0,1, \ldots
\end{aligned}
$$

where $\epsilon$ and $\delta$ are known constants, and $t$ is either 1 or $\infty$. The sequence $\left\{w_{j}\right\}$ may account for the fact that a linear model may not be a sufficiently accurate model of the real system. Thus, the inclusion of this sequence in model (24) provides a degree of robustness to the deterministic estimator proposed. In addition, $\left\{v_{j}\right\}$ may represent measurement noise. This deterministic description is useful when it is only known that the sequence 
$\left\{v_{j}\right\}$ remains bounded as in (27) but no statistical description is known. Also, $\left\{v_{j}\right\}$ may be intentionally included in the system. This is known as dithering (see, e.g., [37]) and may improve the performance of the overall control system.

The state quantization function $\sigma(\cdot)$ in $(28)$ is defined as

$$
\sigma\left(x_{k}\right)=\left\{\begin{array}{ccc}
\sigma_{1}, & \text { if } & x_{k} \in \mathcal{Y}_{1} \\
\sigma_{2}, & \text { if } & x_{k} \in \mathcal{Y}_{2} \\
\vdots & \vdots & \vdots \\
\sigma_{\mathrm{L}}, & \text { if } & x_{k} \in \mathcal{Y}_{\mathrm{L}}
\end{array}\right.
$$

where $\mathrm{L}$ is the number of quantization regions.

At the current time instant, $k$, we would like to combine the information provided by the available measurements in order to obtain the smallest region of state space where the state $x_{k+1}$ is guaranteed to lie. We will henceforth refer to this region as the set-valued state estimate. We make the following assumptions.

A-1) The regions $\mathcal{Y}_{i}, i=1, \ldots, \mathrm{L}$, are dense in $\mathbb{R}^{n}$, nonempty, convex, and polytopic.

A-2) The regions $\mathcal{Y}_{i}, i=1, \ldots, \mathrm{L}$ form a partition of a given convex polytope $\mathcal{H}$, defined in $\mathbb{R}^{n}$.

A-3) $x_{k} \in \mathcal{H}$, for $k=0,1, \ldots$

Remark 6: Assumptions A-2) and A-3) imply that at any time instant, the state will belong to the domain of definition of the quantizer function (28).

Remark 7: Note that the state quantizer (28) is more general than the output quantizer (3). In fact, depending on the choice of regions $\mathcal{Y}_{i}, i=1, \ldots, \mathrm{L}$, the general state quantizer defined in (28) may produce the same measurements as an output quantizer of the form

$$
y_{k}=\sigma_{o}\left(C x_{k}+v_{k}^{o}\right)
$$

Hence, the state quantizer given in (28) incorporates output quantization as a special case.

Remark 8: Many papers in the area of set-valued estimation utilize bounding ellipsoids [21], [22], [38], [39]. Our goal is to obtain the smallest set-valued estimate possible, which, according to the assumptions adopted, can be proven to be polytopic. This has close connections to finding the exact description of the feasible parameter set in bounded-error parameter estimation (see, e.g., [40]), especially for the case of time-varying parameters. Ellipsoidal bounding sets are frequently used since the amount of storage memory needed for a single ellipsoidal set description does not depend on the amount of information (number of measurements) received from the system. On the other hand, the amount of memory needed to store the polytopic set may grow with the number of measurements. In this context, we make a contribution insofar as we limit computational complexity by deploying a block moving horizon strategy.

2) Set-Valued Observer Design: In this section, we develop a set-valued observer for the problem described in the previous section. We also discuss how point state estimates are obtained. For similar reasons as discussed previously in Remark 4, we design the observer so that the information available at time instant $k$ is used to calculate the set-valued estimate for state $x_{k+1}$.

a) Information in the presence of measurement noise: If no measurement noise was present $\left(v_{k} \equiv 0\right)$, the event of receiving the quantized measurement $y_{k}^{q}=\sigma_{i}$ would imply that $x_{k} \in \mathcal{Y}_{i}$ [see (28)]. However, measurement noise could cause a measurement indicating a different region to be received whenever the state $x_{k}$ is closer than $\epsilon$ [see (27)] to the border of region $\mathcal{Y}_{i}$. We overcome this problem in the following way. Whenever a measurement $y_{k}^{q}=\sigma_{i}$ is received, the observer assumes that $x_{k} \in \operatorname{Expand}\left(\mathcal{Y}_{i}, \epsilon\right) \cap \mathcal{H}$, where $\operatorname{Expand}(\cdot, \cdot)$ is defined by

$\operatorname{Expand}(\mathcal{Y}, \epsilon) \triangleq\left\{x \in \mathbb{R}^{n}: x=z+v\right.$, for some $z \in \mathcal{Y}$
and some $v$ such that $\left.\|v\|_{t} \leq \epsilon\right\}$.

If $t=1$ or $\infty$ and $\mathcal{Y}_{i}$ is polytopic, we can readily see that $\operatorname{Expand}\left(\mathcal{Y}_{i}, \epsilon\right)$ also is polytopic. We thus define

$$
\mathcal{Y}_{i}^{\text {OBS }} \triangleq \operatorname{Expand}\left(\mathcal{Y}_{i}, \epsilon\right) \cap \mathcal{H}, \quad i=1, \ldots, \text { L. }
$$

Hence, it is certain that $x_{k} \in \mathcal{Y}_{i}^{\text {OBS }}$ whenever the measurement $y_{k}^{q}=\sigma_{i}$ is received.

Remark 9: The previous region expansion process yields another convex polytope. Hence, this operation preserves both the convexity and the polytopic characteristics of the regions.

b) Obtaining the set-valued estimate: We start by explaining the initialization procedure and how the first iteration is performed. Then, we develop a recursive algorithm to be utilized by the observer at each successive time instant. In the sequel, let $\mathcal{X}_{j+1}$ denote the set-valued state estimate of $x_{j+1}$ based on measurements $y_{0}^{q}, \ldots, y_{j}^{q}$.

Initialization: By assumption $\mathrm{A}-3$ ), the initial state, $x_{0}$, belongs to the region $\mathcal{H}$. Since no additional information is available at time $k=0$, the set-valued estimate at time $k=0$ is simply $\mathcal{X}_{0} \triangleq \mathcal{H}$.

First Iteration: The measurement $y_{0}^{q}=\sigma_{i}$ becomes available. From (30), it is now known that $x_{0} \in \mathcal{Y}_{i}^{\text {OBS }}$. It is also known that $x_{0} \in \mathcal{X}_{0}$. Hence

$$
x_{0} \in \mathcal{Y}_{i}^{\mathrm{OBS}} \cap \mathcal{X}_{0}
$$

We now define the region

$$
\mathcal{X}_{0}^{\mathrm{M}} \triangleq \mathcal{Y}_{i}^{\mathrm{OBS}} \cap \mathcal{X}_{0}
$$

where the superscript ${ }^{M}$ is used to indicate that the region has been updated using the information provided by the last available measurement.

To obtain the set-valued estimate $\mathcal{X}_{1}$, for the state at time instant $k=1$, we must transform the region $\mathcal{X}_{0}^{\mathrm{M}}$ by means of the system model (24). This can be performed in two steps

$$
\begin{gathered}
\tilde{\mathcal{X}}_{1}=\left\{x_{1} \in \mathbb{R}^{n}: x_{1}=A x_{0}+B u_{0},\right. \\
\text { for some } \left.x_{0} \in \mathcal{X}_{0}^{\mathrm{M}}\right\} \\
\mathcal{X}_{1}=\operatorname{Expand}\left(\tilde{\mathcal{X}}_{1}, \delta\right) \cap \mathcal{H} .
\end{gathered}
$$

The region $\mathcal{X}_{1}$ is the set-valued estimate of the state $x_{k+1}$. All available information has already been used in order to calculate it. We will denote the calculation in (33) by

$$
\tilde{\mathcal{X}}_{1}=A \mathcal{X}_{0}^{\mathrm{M}}+B u_{0}
$$

Observer Algorithm: The procedure described previously provides a one-step-ahead set-valued estimate of the state at 
time $k=0$. We describe the procedure at an arbitrary time instant $k>0$ in the following recursive algorithm.

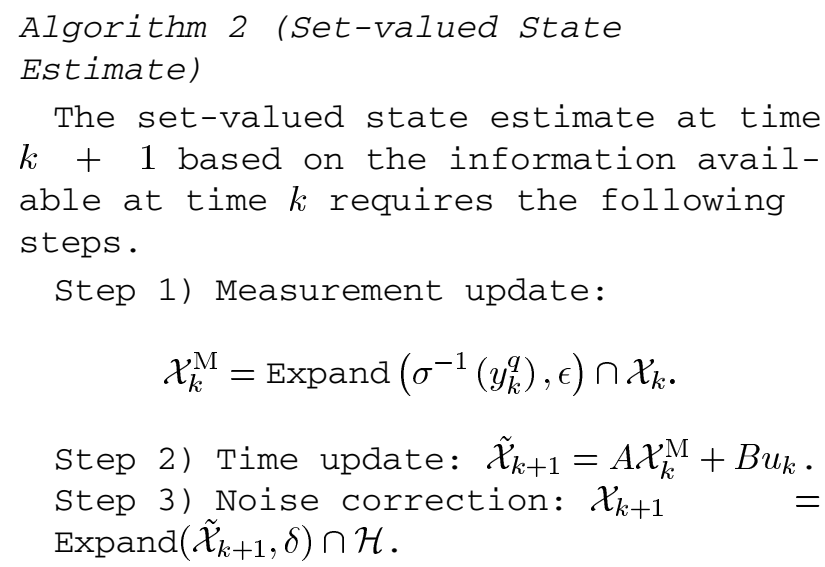

Proposition 1 (Convexity of Set-Valued Estimates): The regions $\mathcal{X}_{k}, k=0,1, \ldots$, calculated by Algorithm 2 are either empty or convex polytopes.

Proof: (By induction) By assumption A-2), $\mathcal{X}_{0}=\mathcal{H}$ is a convex polytope. Now, assume that the region $\mathcal{X}_{k}$ is a convex polytope. By assumption A-1), $\sigma^{-1}\left(y_{k}^{q}\right)$ is also a convex polytope. By Remark 9 and Step 1) of Algorithm 2, it follows that $\mathcal{X}_{k}^{\mathrm{M}}$ is either a convex polytope or empty since it is the result of intersecting two convex polytopes (see, e.g., [41]). From Step 2), $\tilde{\mathcal{X}}_{k+1}$ is obtained via an affine transformation of a convex-polytopic or empty region and, hence, it also must be either convexpolytopic or empty [41]. From Step 3) and Remark 9, the result follows.

Proposition 2 (Empty Set-Valued Estimates): If, during the iteration of Algorithm 2, an empty region is obtained, then all subsequent regions will also be empty.

Proof: (By induction) Assume $\mathcal{X}_{k}=\emptyset$. Then, by Step 1) of Algorithm 2, $\mathcal{X}_{k}^{\mathrm{M}}=\emptyset$. By this and Steps 2) and 3), we have that $\mathcal{X}_{k}=\emptyset \Rightarrow \mathcal{X}_{k+1}=\emptyset$. The result then follows.

c) Dealing with empty set-valued estimates: The occurrence of an empty region as the result of Algorithm 2 implies that the measurements obtained up to that moment could not have been generated by the system defined in (24) and (25) under the assumptions made. This implies that the set-valued observer is unable to supply any useful information regarding the location of the state. We will henceforth assume that this situation can only be caused by what we call impulse-like disturbances. By this terminology, we mean disturbances that occur during one time instant and may have a noticeable effect on the system states. To deal with this situation, we add the following assumption.

A-4) Occasionally, the state is affected by a disturbance that may instantly change the position of the state to any point in the region $\mathcal{H} .{ }^{1}$

We add the following modification to the observer strategy: whenever an empty region is obtained, the observer will reset, discarding all previous information except for the very last measurement, and begin again from that time instant.

\footnotetext{
${ }^{1}$ This is the case when step-like disturbances are modeled as additional states, see the tank level control system in Sections IV and V.
}

By including this modification, we obtain the final version of the observer algorithm proposed in this section.

$$
\begin{aligned}
& \text { Algorithm } 3 \text { (Observer Algorithm-Final } \\
& \text { Version) } \\
& \text { Step 1) Measurement update: } \\
& \qquad \mathcal{X}_{k}^{\mathrm{M}}=\text { Expand }\left(\sigma^{-1}\left(y_{k}^{q}\right), \epsilon\right) \cap \mathcal{X}_{k} \cdot \\
& \text { Step 2) Disturbance detection: } \\
& \text { If } \mathcal{X}_{k}^{\mathrm{M}}=\emptyset \text {, set } \mathcal{X}_{k}^{\mathrm{M}}=\text { Expand }\left(\sigma^{-1}\left(y_{k}^{q}\right), \epsilon\right) . \\
& \text { Step 3) Time update: } \tilde{\mathcal{X}}_{k+1}=A \mathcal{X}_{k}^{\mathrm{M}}+B u_{k} \cdot \\
& \text { Step 4) Noise correction: } \mathcal{X}_{k+1} \\
& \text { Expand }\left(\tilde{\mathcal{X}}_{k+1}, \delta\right) \cap \mathcal{H} .
\end{aligned}
$$

d) Point state estimate: The controller to be developed in Section III requires a point state estimate instead of a set-valued estimate. Whenever the set-valued estimate $\mathcal{X}_{k}$ is nonempty, any point contained in it is a possible value for the state $x_{k}$. We propose to use a Chebyshev center (see [42, Sec. 8.5]) of $\mathcal{X}_{k}$ as the required state estimate. A Chebyshev center is defined as

$$
x_{\text {cheb }}\left(\mathcal{X}_{k}\right)=\underset{x \in \mathcal{X}_{k}}{\operatorname{argmax}} \operatorname{dist}\left(x, \mathbb{R}^{n} \backslash \mathcal{X}_{k}\right)
$$

where $\operatorname{dist}\left(x, \mathbb{R}^{n} \backslash \mathcal{X}_{k}\right)$ denotes the Euclidean distance from the point $x$ to the set $\mathbb{R}^{n} \backslash \mathcal{X}_{k}$.

Remark 10: Note that, by definition, a Chebyshev center of a convex region is a point that belongs to the region and may not be unique.

3) Computational Issues: Computational complexity may increase in every iteration of the algorithm due to the need for intersecting polytopes, i.e., the polytope $\mathcal{X}_{k+1}$ may contain a higher number of vertices than $\mathcal{X}_{k}$. Thus, a crucial aspect in the computational implementation of Algorithm 3 is to bound the complexity of the polytopic regions.

We propose to limit computational complexity by deploying a block moving horizon strategy. This strategy consists in running $\mathrm{N}_{\mathrm{O}}$ parallel observers at every time instant and combining them in a moving horizon manner (see Fig. 2). As in the stochastic estimator developed in Section II-A, previous measurements are suitably summarized. The summarizing step is carried out by bounding the polytopic region obtained with an axis-aligned hypercube. This bounding step is responsible for reducing the worst-case complexity of all the polytopic regions obtained. In Fig. 2, each observer bounds the polytopic region obtained after running for a period of $\mathrm{N}$ time instants. At any time instant, all observers calculate a set-valued estimate. The required set-valued estimate is provided by the observer that performed the region-bounding step the longest time ago. In Fig. $2, \mathcal{X}_{k_{1}+1}$ is provided, at time $k_{1}$, by observer number 1 whereas at time $k_{2}$, observer number 2 provides $\mathcal{X}_{k_{2}+1}$. We can see that the lowest number of measurements that are explicitly taken into account is $\mathrm{N}-\mathrm{N} / \mathrm{N}_{\mathrm{O}}=\mathrm{N} \cdot\left(\mathrm{N}_{\mathrm{O}}-1\right) / \mathrm{N}_{\mathrm{O}}$ (except at startup, when not enough measurements have been taken), and this happens when the estimate is provided one time instant after one of the observers has performed the region bounding step. Note that the parameters $\mathrm{N}$ and $\mathrm{N}_{\mathrm{O}}$ allow one to tradeoff observer 


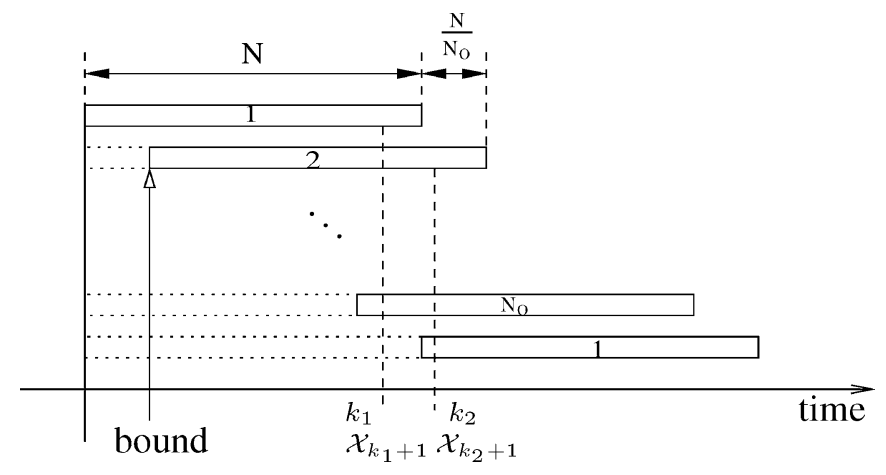

Fig. 2. Block moving horizon strategy.

performance for computational complexity. Higher values of the parameter $\mathrm{N}$ yield better performance since the maximum number of measurements that are explicitly considered becomes higher, but the computational complexity may also be higher since the polytopes may potentially contain a larger number of vertices. In this sense, it may be said that $\mathrm{N}$ provides an upper bound on the performance. On the other hand, the number of observers running in parallel is responsible for a lower bound on the performance, i.e., the number of measurements explicitly considered is always greater than $\mathrm{N} \cdot\left(\mathrm{N}_{\mathrm{O}}-1\right) / \mathrm{N}_{\mathrm{O}}$. Computational complexity in this case is multiplied by a factor $\mathrm{N}_{\mathrm{O}}$, since we have $\mathrm{N}_{\mathrm{O}}$ observers running in parallel at every instant. If $\mathrm{N}_{\mathrm{O}}=\mathrm{N}=\mathrm{N}_{\mathrm{M}}$, the suggested strategy becomes the exact deterministic counterpart to the stochastic observer designed in Section II-A.

\section{DOWN-LINK DESIGN}

We next consider the down-link problem. For that purpose we treat the (point) state estimates provided by the methodology of Section II as if they were the true states (i.e., we apply certainty equivalence). We show that communication constraints which arise in the networked control system architecture can be reduced to a particular finite set constraint on the signals to be sent to the actuators. As a consequence, the down-link design procedure fits into the finite-set constrained control setting presented in [28]. However, in order to apply the results of [28], we need to extend the framework to multivariable systems and to the case of nonzero set-points. A further contribution is contained in Section III-F, where a scheme which ensures closed-loop stability under state feedback is proposed.

\section{A. Problem Statement}

As foreshadowed in Section I, we assume that the link between the controller and actuators is characterized by a known and fixed time-delay and that data is sent at a bounded rate. This is achieved by imposing the following two communication constraints on the design.

Constraint 1: The data sent from the controller to each actuator is restricted to belong to a (small and fixed) finite set of scalars, $\mathbb{U}$.

Constraint 2: Only data corresponding to one input of the plant can be transmitted at a time. Between updates, (which may be separated by several sampling periods) all plant inputs are held at their previous values.

The delay between controller and plant can be incorporated into the multiple-input-multiple-output noiseless plant model

$$
\begin{aligned}
x_{j+1} & =A x_{j}+B u_{j} \\
y_{j} & =C x_{j}
\end{aligned}
$$

where $x_{j} \in \mathbb{R}^{n}, u_{j} \in \mathbb{R}^{m}, y_{j} \in \mathbb{R}^{s}, A \in \mathbb{R}^{n \times n}, B \in \mathbb{R}^{n \times m}$, and $C \in \mathbb{R}^{s \times n}$, compare to (24). For future reference, we note that $u_{j}$ can be expanded as

$$
u_{j}=\left[\begin{array}{llll}
\left(u_{1}\right)_{j} & \left(u_{2}\right)_{j} & \cdots & \left(u_{m}\right)_{j}
\end{array}\right]^{T} .
$$

We assume that we are given a constant set-point vector $r^{\star}$. The design problem can thus be stated as that of developing a control strategy which regulates the model (36) to $r^{\star}$, while not violating constraints 1 or 2 . Thus, the control strategy for the networked system is characterized by choosing, at each time step, which of the $m$ inputs in (37) to access and what to send, i.e., the controller needs to divide its attention between all plant inputs.

In the following section, we translate this design problem into an optimal control framework.

Remark 11 (Control of Multiple Plants): The previous formulation encompasses the problem of controlling a collection of geographically separate plants, in which case (36) represents the entire set of plants.

\section{B. Specification of the Down-Link}

Rather than sending the control signals directly, we propose to send their increments:

$$
\Delta\left(u_{i}\right)_{k} \triangleq\left(u_{i}\right)_{k}-\left(u_{i}\right)_{k-1}
$$

when nonzero. This choice, which is inspired by principles of Delta-Modulation (see, e.g., [36, Ch. 3]) generally requires less bits to specify the control signal. The pair $\left(\Delta\left(u_{i}\right)_{k}, i\right)$ is received at the actuator node specified by the index $i$. The actual input signal $\left(u_{i}\right)_{k}$ is readily reconstructed by discrete-time integration as shown in Fig. 3.2

Communication constraints 1 and 2 can be summarized by means of a simple finite-set constraint on the increments (38). More precisely, at every time instant $k$, the vector

$$
\Delta u_{k} \triangleq\left[\begin{array}{llll}
\Delta\left(u_{1}\right)_{k} & \Delta\left(u_{2}\right)_{k} & \ldots & \Delta\left(u_{m}\right)_{k}
\end{array}\right]^{T}
$$

is restricted to belong to the set $\mathbb{V}$, which is defined as

$$
\mathbb{V} \triangleq\left\{\eta \in \mathbb{R}^{m}: \eta=\left[\begin{array}{lllll}
0 \ldots 0 & \nu & 0 & \ldots
\end{array}\right]^{T}, \text { for some } \nu \in \mathbb{U}\right\} \text {. }
$$

As depicted in Fig. 4, this set contains all column vectors formed by one element of $\mathbb{U}$, while all its other components are zero.

Remark 12 (Design of the Coder-Decoder Pair): In (38), we propose to send coded actuator data in form of increments and to integrate (decode) the received data at the actuator node, see Fig. 3. This contrasts with other strategies in which the

${ }^{2} \rho$ denotes the forward shift operator, $\rho v_{k}=v_{k+1}$, where $\left\{v_{k}\right\}$ is any sequence. 


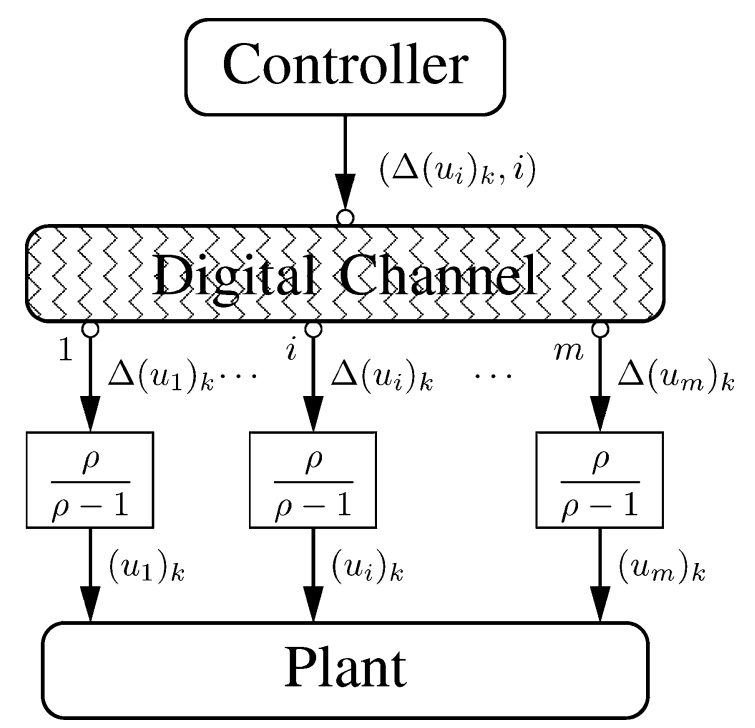

Fig. 3. Down-link design.

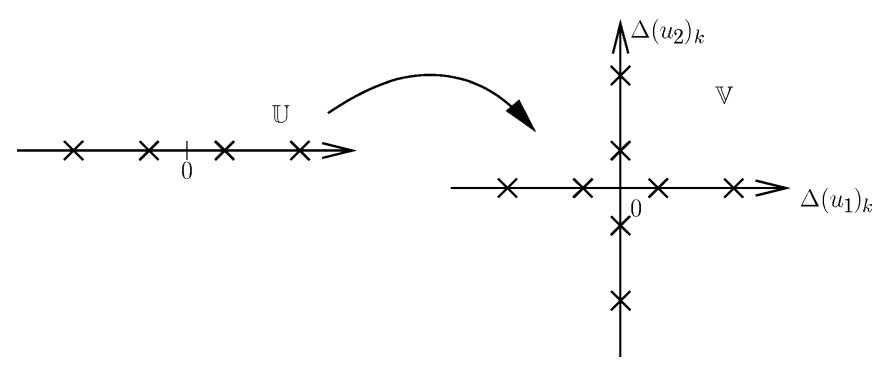

Fig. 4. Construction of the set $\mathbb{V}$.

inputs are reset to zero after each sample period, see, e.g., [43]. While the choice of coder-decoder pair given in (38) allows one to reduce down-link network traffic significantly, there may also be some drawbacks. Indeed, due to the integrating effect of the decoder, the effect of undesired channel disturbances, such as missing and/or corrupted data, will, in principle, propagate indefinitely in time. As a consequence, the controller may lose track of the actual signal applied to the plant inputs, leading to performance degradation. In order to avoid this problem, one can feed back the plant inputs, at the expense of additional up-link traffic. Alternatively, it is also possible to deploy more general coder-decoder pairs. As shown in [44], a design problem can be formulated that trades off down-link traffic versus immunity to channel disturbances.

\section{Performance Measure}

The performance of the model (36) over a finite horizon $\mathrm{N}_{\mathrm{P}}$ starting at time $k$ can be quantified by means of the quadratic $\operatorname{cost}^{3}$

$$
V_{\mathrm{N}_{\mathrm{P}}} \triangleq \sum_{j=k+1}^{k+\mathrm{N}_{\mathrm{P}}}\left\|y_{j}^{\prime}-r^{\star}\right\|^{2}+\sum_{j=k}^{k+\mathrm{N}_{\mathrm{P}}-1}\left\|u_{j}-u^{\star}\right\|_{R}^{2}
$$

\footnotetext{
${ }^{3}$ In the sequel we will use $\|y\|$ to denote the Euclidean norm $\sqrt{y^{T} y}$, and $\|u\|_{R}$ to denote the weighted norm, $\sqrt{u^{T} R u}$.
}

where $R=R^{T}>0$ and $u^{\star}$ is an input that produces $r^{\star}$ in steady state, i.e., satisfies

$$
r^{\star}=C\left(I_{n}-A\right)^{-1} B u^{\star} .
$$

In this cost, $y_{j}^{\prime}$ and $u_{j}$ are predicted trajectories that follow the prediction model

$$
\begin{aligned}
x_{j+1}^{\prime} & =A x_{j}^{\prime}+B u_{j}, \quad j \geq k \\
y_{j}^{\prime} & =C x_{j}^{\prime}
\end{aligned}
$$

with initial condition $x_{k}^{\prime}=x_{k}$, the current state.

Remark 13 (The Role of $R$ ): Note that the matrix $R$ in the performance measure (39) penalizes transmission rates to the actuators selectively. This is particularly useful in the NCS setting considered, where some actuators may be easier to access than others. The choice of $R$ can be made by taking into account different levels of congestion and transmission quality of the communication routes.

As explained in Section III-B, control increments $\Delta u_{j}$ are to be sent down the channel. Thus, the decision variables of the cost (39) are the predicted control increments

$$
\Delta u_{j} \triangleq u_{j}-u_{j-1}, \quad j=k, \ldots, k+\mathrm{N}_{\mathrm{P}}-1 .
$$

These can be gathered into the vector

$$
\begin{aligned}
& \Delta \overrightarrow{\mathbf{u}}_{k: k+\mathrm{N}_{\mathrm{P}}-1} \\
& \quad \triangleq\left[\left(\Delta u_{k}\right)^{T}\left(\Delta u_{k+1}\right)^{T} \ldots\left(\Delta u_{k+\mathrm{N}_{\mathrm{P}}-1}\right)^{T}\right]^{T} \in \mathbb{R}^{\mathrm{N}_{\mathrm{P}} \cdot m}
\end{aligned}
$$

which needs to satisfy the finite set constraint

$$
\Delta \overrightarrow{\mathbf{u}}_{k: k+N_{\mathrm{P}}-1} \in \mathbb{V}^{N_{\mathrm{P}}}
$$

where

$$
\mathbb{V}^{N_{P}} \triangleq \mathbb{V} \times \cdots \times \mathbb{V}
$$

In the remainder of this section, we will write $V_{\mathrm{N}_{\mathrm{P}}}\left(\Delta \overrightarrow{\mathbf{u}}_{k: k+\mathrm{N}_{\mathrm{P}}-1}\right)$ in order to make this dependence explicit. Note that the cost function (39) also depends upon the values of $x_{k}$ and $u_{k-1}$. Both quantities are assumed to be known for the purpose of calculating the control law.

Remark 14 (Magnitude Constraints): The present framework can be extended to plants whose inputs are affected by saturation. In this case, the predicted increments $\Delta \overrightarrow{\mathbf{u}}_{k: k+N_{\mathrm{P}}-1}$ can be restricted to belong to a subset of $\mathbb{V}^{N_{P}}$, which keeps predicted inputs $u_{k+i}, i=0, \ldots, \mathrm{N}_{\mathrm{P}}-1$ within the unsaturated region. Although the resulting subsets are time-varying, since they depend upon the value of the previous control input, $u_{k-1}$, it can readily be seen that the inclusion of magnitude constraints presents no conceptual difficulties.

\section{Receding Horizon Formulation}

Based upon the cost (39), we propose to utilize a receding horizon scheme as deployed in model predictive control; see, 
e.g., [16]. Therefore, at each time step, we solve the finite-set constrained quadratic program

$$
\Delta \overrightarrow{\mathbf{u}}_{k: k+\mathrm{N}_{\mathrm{P}}-1}^{\mathrm{opt}} \triangleq \arg \min _{\Delta \overrightarrow{\mathbf{u}}_{k: k+\mathrm{N}_{\mathrm{P}}-1} \in \mathbb{V}^{\mathrm{N}_{\mathrm{P}}}} V_{\mathrm{N}_{\mathrm{P}}}\left(\Delta \overrightarrow{\mathbf{u}}_{k: k+\mathrm{N}_{\mathrm{P}}-1}\right) .
$$

Instead of implementing the entire control sequence contained in $\Delta \overrightarrow{\mathbf{u}}_{k: k+\mathrm{N}_{\mathrm{P}}-1}^{\mathrm{opt}}$, we only utilize its first $m$ components

$$
\Delta u_{k}^{\mathrm{opt}} \triangleq L^{T} \Delta \overrightarrow{\mathbf{u}}_{k: k+\mathrm{N}_{\mathrm{P}}-1}^{\mathrm{opt}}
$$

where

$$
L \triangleq\left[\begin{array}{llll}
I_{m} & 0_{m} & \ldots & 0_{m}
\end{array}\right]^{T} \in \mathbb{R}^{\mathrm{N}_{\mathrm{P}} \cdot m \times m}
$$

and $0_{m} \triangleq 0 \cdot I_{m}$.

This vector contains the data corresponding to all $m$ inputs. Since $\Delta u_{k}^{\text {opt }} \in \mathbb{V}$, not more than one of its components is nonzero. Only this value is sent to the plant input determined by its index, see Fig. 3. The other $m-1$ inputs are left unchanged as prescribed in Constraint 2. It can be seen that the strategy only sends the most relevant control increment, as quantified by the cost (39).

We will call the networked controller specified by (45) and (46) a model predictive networked controller (MPNC). It makes decisions based upon overall future performance of the multivariable plant(s) which respect constraints 1 and 2 . Note that not only the value of the control increment to be applied at each time is provided by solving (45) but also the question of which input to access is answered. Thus, the MPNC yields the optimal communication sequence (borrowing terminology from [26]; see also [27]).

\section{E. Implementation of the MPNC}

In this section, we develop a "closed-form" solution to the optimization problem (45). As will be apparent, the MPNC can be implemented by utilizing linear filters and a vector quantizer.

For that purpose, it is useful to define the vectors

$$
\begin{aligned}
\overrightarrow{\mathbf{u}}_{k: k+\mathrm{N}_{\mathrm{P}}-1} \triangleq\left[\begin{array}{c}
u_{k} \\
u_{k+1} \\
\vdots \\
u_{k+\mathrm{N}_{\mathrm{P}}-1}
\end{array}\right] \quad \overrightarrow{\mathbf{y}}_{k+1: k+\mathrm{N}_{\mathrm{P}}}^{\prime} \triangleq\left[\begin{array}{c}
y_{k+1}^{\prime} \\
y_{k+2}^{\prime} \\
\vdots \\
y_{k+\mathrm{N}_{\mathrm{P}}}^{\prime}
\end{array}\right] \\
\overrightarrow{\mathbf{r}}^{\star} \triangleq\left[\begin{array}{c}
r^{\star} \\
r^{\star} \\
\vdots \\
r^{\star}
\end{array}\right] \quad \overrightarrow{\mathbf{u}}^{\star} \triangleq\left[\begin{array}{c}
u^{\star} \\
u^{\star} \\
\vdots \\
u^{\star}
\end{array}\right]
\end{aligned}
$$

and to note that (39) can be rewritten in vector form as

$$
\begin{aligned}
& V_{\mathrm{N}_{\mathrm{P}}}\left(\Delta \overrightarrow{\mathbf{u}}_{k: k+\mathrm{N}_{\mathrm{P}}-1}\right) \\
& \quad=\left\|\overrightarrow{\mathbf{y}}_{k+1: k+\mathrm{N}_{\mathrm{P}}}^{\prime}-\overrightarrow{\mathbf{r}}^{\star}\right\|^{2}+\left\|\overrightarrow{\mathbf{u}}_{k: k+\mathrm{N}_{\mathrm{P}}-1}-\overrightarrow{\mathbf{u}}^{\star}\right\|_{R}^{2}
\end{aligned}
$$

with $\bar{R} \triangleq \operatorname{diag}(R, \ldots, R)$. Furthermore, iteration of (41) yields

$$
\overrightarrow{\mathbf{y}}_{k+1: k+\mathrm{N}_{\mathrm{P}}}^{\prime}=\Phi_{\mathrm{N}_{\mathrm{P}}} \overrightarrow{\mathbf{u}}_{k: k+\mathrm{N}_{\mathrm{P}}-1}+\Lambda_{\mathrm{N}_{\mathrm{P}}} x_{k}
$$

where

$$
\begin{aligned}
& \Phi_{\mathrm{N}_{\mathrm{P}}} \triangleq {\left[\begin{array}{cccc}
C B & 0 & \ldots & 0 \\
C A B & C B & \ddots & \vdots \\
\vdots & \ddots & \ddots & 0 \\
C A^{\mathrm{N}_{\mathrm{P}}-1} B & \ldots & C A B & C B
\end{array}\right] } \\
& \Lambda_{\mathrm{N}_{\mathrm{P}}} \triangleq\left[\begin{array}{c}
C A \\
C A^{2} \\
\vdots \\
C A^{\mathrm{N}_{\mathrm{P}}}
\end{array}\right] .
\end{aligned}
$$

(Note that, to simplify the notation we drop the subscript $N_{P}$ in the sequel and write $\Phi$ and $\Lambda$ to denote $\Phi_{N_{P}}$ and $\Lambda_{N_{P}}$, respectively.)

In order to include the predicted increments of (43) in these expressions, notice that

$$
\Delta \overrightarrow{\mathbf{u}}_{k: k+\mathrm{N}_{\mathrm{P}}-1}=K \overrightarrow{\mathbf{u}}_{k: k+\mathrm{N}_{\mathrm{P}}-1}-L u_{k-1}
$$

where $K \triangleq I_{\mathrm{N}_{\mathrm{P}} \cdot m}-M$ and

$$
M \triangleq\left[\begin{array}{ccccc}
0_{m} & \ldots & & \ldots & 0_{m} \\
I_{m} & 0_{m} & & & \vdots \\
0_{m} & \ddots & \ddots & & \\
\vdots & \ddots & \ddots & \ddots & \vdots \\
0_{m} & \ldots & 0_{m} & I_{m} & 0_{m}
\end{array}\right] \in \mathbb{R}^{\mathrm{N}_{\mathrm{P}} \cdot m \times \mathrm{N}_{\mathrm{P}} \cdot m}
$$

and $L$ is defined in (47). Note that $K$ is invertible.

Given these relations, the MPNC which yields the receding horizon law (46) can be characterized in a simple fashion as described in Theorem 1. It makes use of a nearest neighbor vector quantizer, defined as follows.

Definition 1 (Nearest Neighbor Vector Quantizer): Given a countable set of nonequal vectors $\mathcal{B}=\left\{b_{1}, b_{2}, \ldots\right\} \subset \mathbb{R}^{n_{\mathcal{B}}}$, the nearest neighbor quantizer is defined as a mapping $q_{\mathcal{B}}: \mathbb{R}^{n_{\mathcal{B}}} \rightarrow$ $\mathcal{B}$ which assigns to each vector $c \in \mathbb{R}^{n_{\mathcal{B}}}$ the closest element of $\mathcal{B}$ (as measured by the Euclidean norm), i.e., $q_{\mathcal{B}}(c)=b \in \mathcal{B}$ if and only if $b$ satisfies: $\|c-b\| \leq\left\|c-b_{i}\right\|, \forall b_{i} \in \mathcal{B}$.

(Note that the aforementioned definition is a special case of (28). A thorough treatment of vector quantizers and their features can be found in the book [45].)

This definition allows us to state the following result.

Theorem 1 (Closed-Form Solution): The optimizer $\Delta \overrightarrow{\mathbf{u}}_{k: k+\mathrm{N}_{\mathrm{P}}-1}^{\mathrm{opt}}$ in (45) satisfies

$$
\Delta \overrightarrow{\mathbf{u}}_{k: k+\mathrm{N}_{\mathrm{P}}-1}^{\mathrm{opt}}=W^{-\frac{1}{2}} q_{\widetilde{\mathbb{V}}_{\mathrm{N} P}}\left(-\left(W^{-\frac{1}{2}}\right)^{T} F\right)
$$

where

$$
\begin{aligned}
& W \triangleq\left(K^{-1}\right)^{T}\left(\Phi^{T} \Phi+\bar{R}\right) K^{-1}>0 \\
& F \triangleq W L u_{k-1}-\left(K^{-1}\right)^{T}\left(\bar{R} \overrightarrow{\mathbf{u}}^{\star}+\Phi^{T}\left(\overrightarrow{\mathbf{r}}^{\star}-\Lambda x_{k}\right)\right)
\end{aligned}
$$

and the square matrix $W^{1 / 2}$ is defined implicitly via $\left(W^{1 / 2}\right)^{T} W^{1 / 2}=W$. 


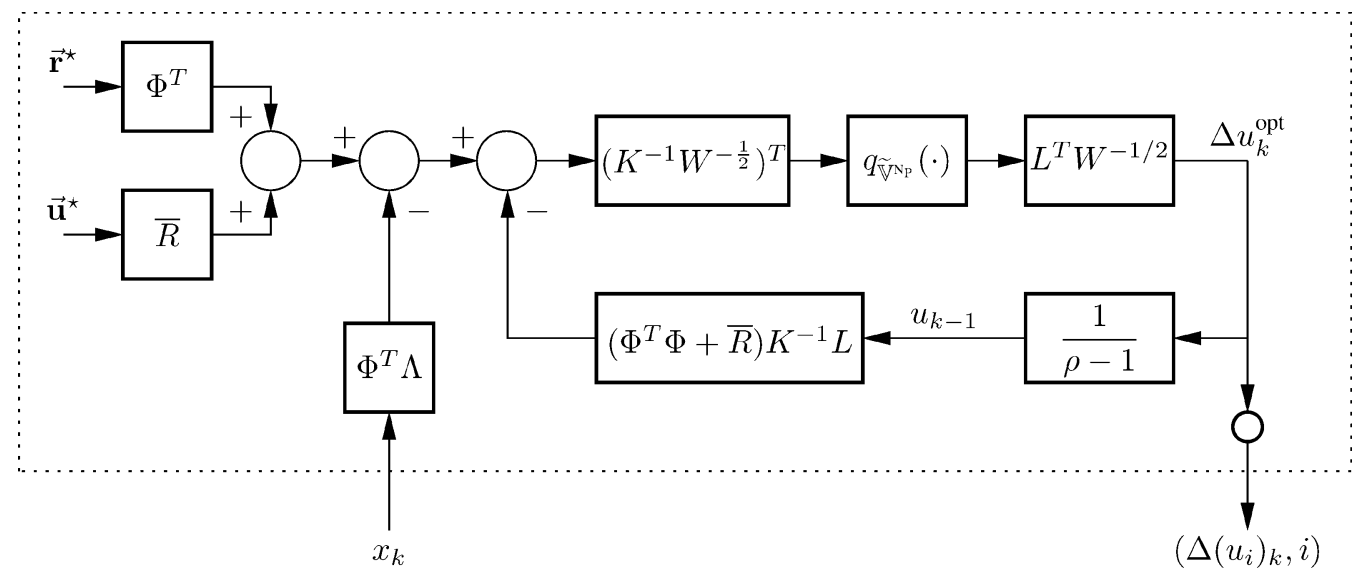

Fig. 5. Implementation of the MPNC.

The nonlinearity $q_{\widetilde{V}_{\mathrm{N}}}(\cdot)$ is a nearest neighbor quantizer as described in Definition 1. The image of this mapping is the set

$$
\widetilde{\mathbb{V}}^{\mathrm{N}_{\mathrm{P}}} \triangleq\left\{\widetilde{V} \in \mathbb{R}^{\mathrm{N}_{\mathrm{P}} \cdot m}: \widetilde{V}=W^{\frac{1}{2}} V \text {, for some } V \in \mathbb{V}^{\mathrm{N}_{\mathrm{P}}}\right\}
$$

i.e., $\widetilde{\mathbb{V}}^{\mathbb{N}}$ is obtained by transforming the (elements of) the set $\mathbb{V}^{N_{P}}$ defined in (44).

Proof: The proof follows similar arguments to those used in the result stated in [28]. Note that care must be taken with (50).

As a consequence of this result and (46), the MPNC can be characterized by means of the closed loop depicted in Fig. 5. As can be appreciated in this figure, the controller corresponds to a time-invariant structure comprising linear filtering and a vector quantizer. Note also, that $\overrightarrow{\mathbf{u}}^{\star}$ and $\overrightarrow{\mathbf{r}}^{\star}$ are related via (40).

Not surprisingly, the MPNC, being a controller which is based upon the minimization of a quadratic cost function, is related to a standard LQR-controller. However, we emphasize here that $\Delta u_{k}^{\mathrm{opt}}$ is, in general, not obtained by nearest neighbor quantization of the solution to an (unconstrained) quadratic programming problem. Indeed, (51) shows that the control signal is calculated by first mapping the term $-\left(W^{-1 / 2}\right)^{T} F$ to the set $\widetilde{\mathbb{V}}^{N_{P}}$ defined in (53). The result of this operation is then multiplied by $W^{-1 / 2}$ yielding a sequence which belongs to the allowed set $\mathbb{V}^{N_{P}}$, see (44). Thus, one can interpret the MPNC as an LQR-related controller, where control signals are constrained to belong to $\mathbb{V}$ (see Fig. 4) in order to meet constraints 1 and 2. Rather than using some ad hoc quantization procedure, the control signals are obtained in an optimal manner, as measured by criterion (39).

\section{F. A Stabilizing Modification of the MPNC}

Ensuring asymptotic stability of the MPNC is, to say the least, nontrivial, due to the communication constraints imposed. In this section, we will enhance the MPNC algorithm in order to obtain asymptotic stability results for the case when the true plant state is known. ${ }^{4}$ Toward this goal, we introduce the cost function

$$
\begin{aligned}
J_{\mathrm{N}_{\mathrm{P}}}\left(\Delta \overrightarrow{\mathbf{u}}_{k: k+\mathrm{N}_{\mathrm{P}}-1}\right) & \triangleq\left\|x_{k+\mathrm{N}_{\mathrm{P}}}^{\prime}-x^{\star}\right\|_{P}^{2} \\
& +\sum_{j=k+1}^{k+\mathrm{N}_{\mathrm{P}}}\left\|y_{j}^{\prime}-r^{\star}\right\|^{2}+\sum_{j=k}^{k+\mathrm{N}_{\mathrm{P}}-1}\left\|u_{j}-u^{\star}\right\|_{R}^{2}
\end{aligned}
$$

with $P=P^{T} \geq 0$ and $R=R^{T}>0$. The vector $x^{\star}$ is the target state, defined as

$$
x^{\star} \triangleq\left(I_{n}-A\right)^{-1} B u^{\star}
$$

which, following (40), yields the desired reference

$$
r^{\star}=C x^{\star} .
$$

While in many MPC schemes (see, e.g., the discussion of [17] or the results for finite set constraints included in [28]) the inclusion of terminal state weighting via the term $\left\|x_{k+\mathrm{N}_{\mathrm{P}}}^{\prime}-x^{\star}\right\|_{P}^{2}$ is sufficient in order to ensure asymptotic stability, the communication constraints imposed, make the networked situation under study more involved.

To obtain stability results, we incorporate an additional constraint in the optimization. Specifically, at each time step, we solve the quadratic program

$$
\Delta \overrightarrow{\mathbf{u}}_{k: k+N_{\mathrm{P}}-1}^{\mathrm{opt}} \triangleq \arg \min J_{\mathrm{NP}_{\mathrm{P}}}\left(\Delta \overrightarrow{\mathbf{u}}_{k: k+\mathrm{N}_{\mathrm{P}}-1}\right)
$$

subject to the finite set constraints:

$$
\begin{aligned}
\Delta \overrightarrow{\mathbf{u}}_{k: k+N_{\mathrm{P}}-1} & \in \mathbb{V}^{N_{\mathrm{P}}} \\
u^{\star}-u_{k+\mathrm{N}_{\mathrm{P}}-1} & \in \mathbb{V} .
\end{aligned}
$$

The constraint (58) refers to the predicted plant input values, rather than their increments. This terminal control constraint (compare to the usage of standard terminal state constraints; see, e.g., [17]), allows one to obtain the following result.

${ }^{4}$ We note that this is clearly an idealizing situation. The more general case of unknown states within this context is a challenging open problem at this point in time. 

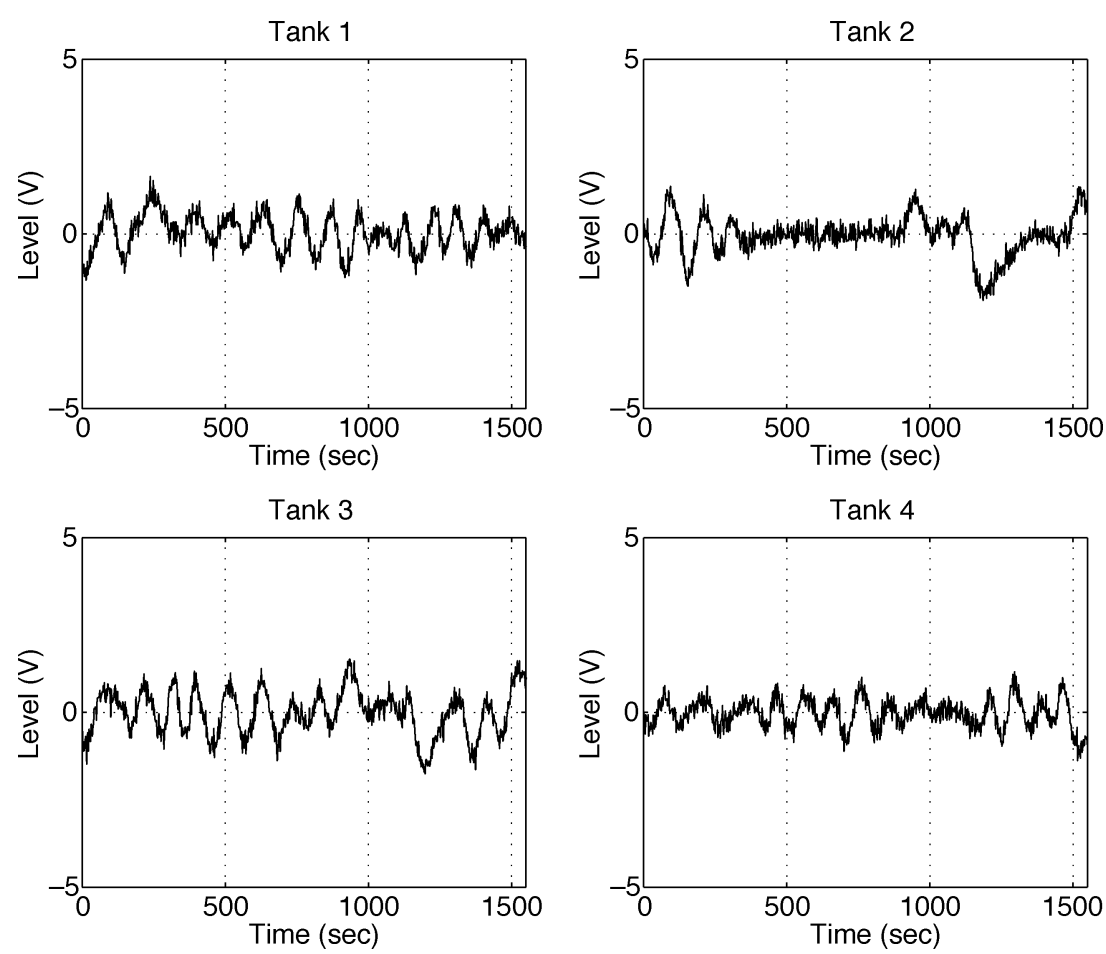

Fig. 6. Actual levels and dithering signal.
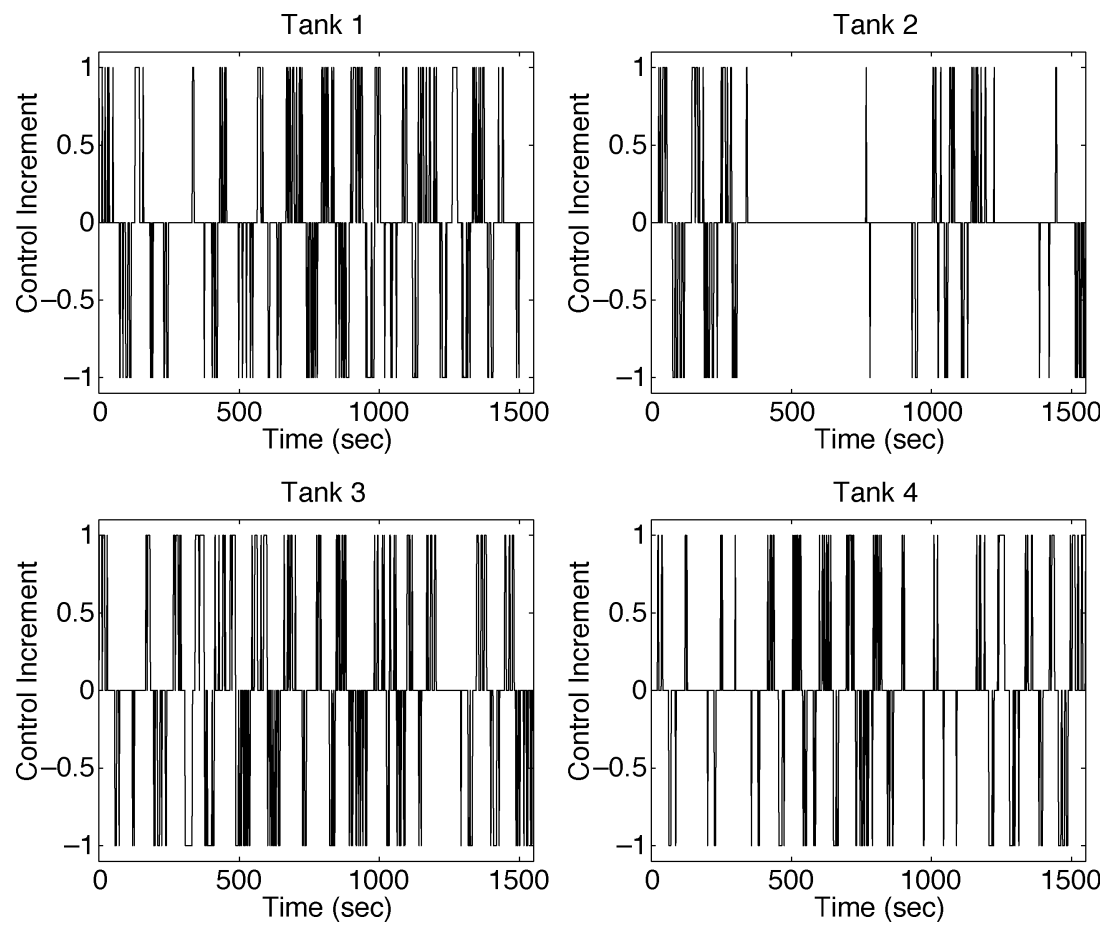

Fig. 7. Control increments.

Theorem 2 (Stability): Consider a stable and observable plant model (36) and suppose that its state is exactly known. If $P$ is chosen to satisfy the discrete-time Lyapunov equation

$$
P=A^{T} P A+A^{T} C^{T} C A
$$

then the receding horizon implementation of (56)-(58) ensures that the plant state $x_{k}$ converges to the target state $x^{\star}$ as $k \rightarrow \infty$.
Proof: The proof is included in the Appendix.

Remark 15 (Achievable Targets): Iteration of (42) with $k=$ 0 yields $u_{\mathrm{N}_{\mathrm{P}}-1}=u_{-1}+\sum_{j=0}^{\mathrm{NP}_{\mathrm{P}}-1} \Delta u_{j}$, where each of the $\Delta u_{j}$ is restricted to belong to $\mathbb{V}$. Thus, the constraint (58) can be rewritten as

$$
u^{\star}-\left(u_{-1}+\sum_{j=0}^{\mathrm{N}_{\mathrm{P}}-1} \Delta u_{j}\right) \in \mathbb{V}
$$



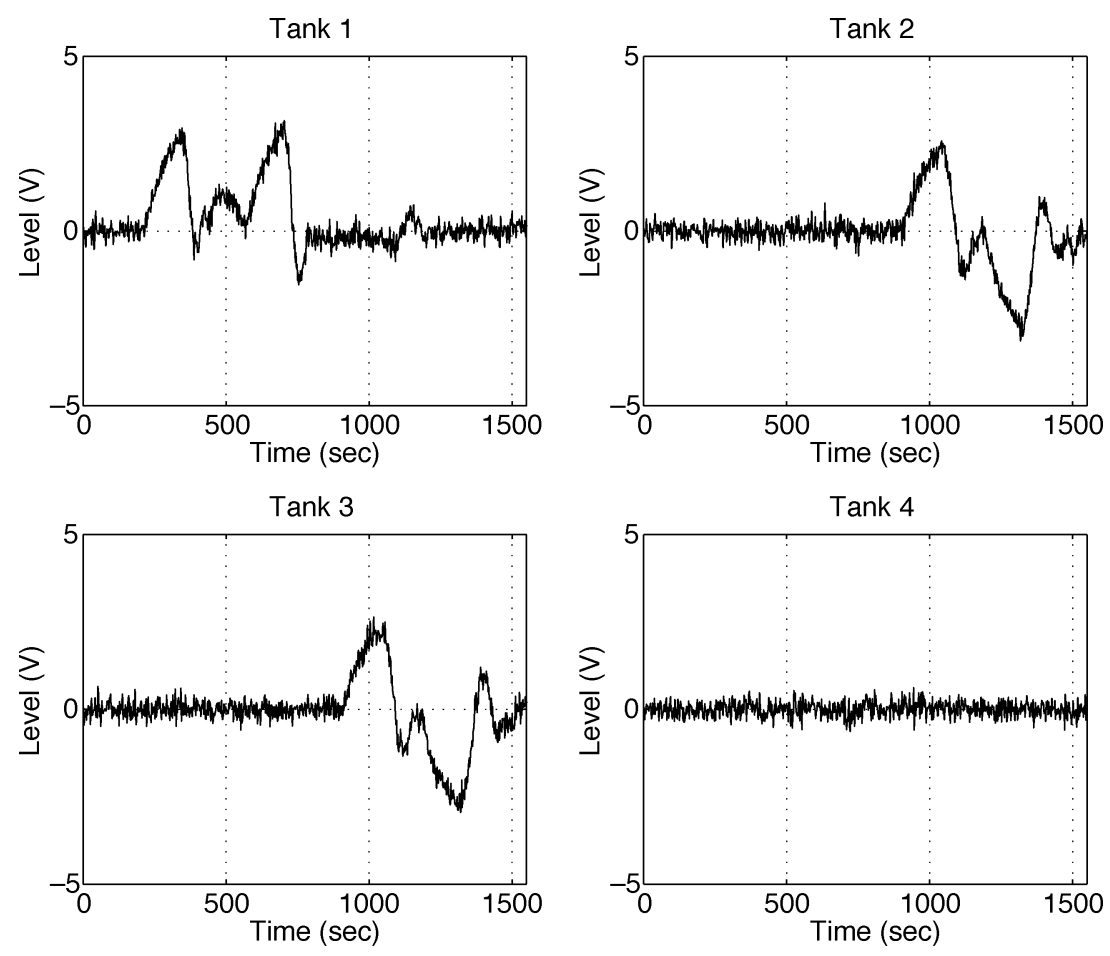

Fig. 8. Actual levels and dithering signal.

with $\Delta u_{j} \in \mathbb{V}, j=0,1, \ldots, \mathrm{N}_{\mathrm{P}}-1$. This means that $u^{\star}$ needs to be expressible as the sum of the initial plant input, $u_{-1}$, and $\mathrm{N}_{\mathrm{P}}$ elements of $\mathbb{V}$. Equivalently, $u^{\star}$ is restricted to belong to the finite set $\mathbb{W}$ defined as ${ }^{5}$

$\begin{aligned} \mathbb{W} \triangleq\left\{\mu \in \mathbb{R}^{m}:\right. & \left(\left(\mu-u_{-1}\right)-\sum_{j=0}^{N_{\mathrm{P}}-1} \eta_{j}\right) \in \mathbb{V}, \\ & \left.\text { for some } \eta_{j} \in \mathbb{V}, j=0,1, \ldots, \mathrm{N}_{\mathrm{P}}-1\right\} .\end{aligned}$

As a consequence of (40), this also restricts the achievable target references of the modified MPNC to belong to a quantized set, namely $r^{\star} \in \mathbb{T}$, with

$$
\mathbb{T} \triangleq\left\{r \in \mathbb{R}^{s}: r=C\left(I_{n}-A\right)^{-1} B u, \text { for some } u \in \mathbb{W}\right\} \text {. }
$$

\section{Simulation Results}

In order to illustrate the main characteristics of the MPNC, we consider level control of four similar tanks. We study both the up-link and down-link problems of controlling the plants over a limited bandwidth channel via a computer simulation of the entire system. We perform two different simulations: the first one utilizes the MC estimator whereas the deterministic estimator is used in the second one.

In each of the four tanks, we seek to maintain a constant level by controlling the voltage applied to an inlet pump. Each tank possesses an orifice of constant size, which is responsible for providing an outflow depending on the height of fluid in the tank. Control is required to reject disturbances from additional inflows and outflows, caused by two disturbing pumps. In our

${ }^{5}$ Structural properties of similar sets have been studied, e.g., in [46]. setup, a single tank having the fluid level as output and, as input, the pump voltage, is described (at the chosen operating point) by the discrete-time model matrices

$$
A_{i}=\left[\begin{array}{cc}
0.9826 & 0.0452 \\
0 & 1
\end{array}\right] \quad B_{i}=\left[\begin{array}{c}
0.0452 \\
0
\end{array}\right] C_{i}=\left[\begin{array}{ll}
1 & 0
\end{array}\right]
$$

where $i=1, \ldots, 4$, for a sampling period of $2 \mathrm{~s}$. The first and second state vector components represent the (incremental) fluid level and disturbance flow around the chosen operating point, respectively. For the controller, the system model (36) is defined as

$A=\operatorname{diag}\left(A_{i}\right) B=\operatorname{diag}\left(B_{i}\right) C=\operatorname{diag}\left(C_{i}\right), \quad i=1, \ldots, 4$.

On the up-link, we transmit only one bit of information per tank, corresponding to whether the tank level is above or below the chosen operating point. ${ }^{6}$ Since we have four tanks and a 2-s sampling period, we need only send $4 \mathrm{~b}$ over a 2 -s period, i.e., the required up-link data rate is $2 \mathrm{~b} / \mathrm{s}$.

On the down-link side, we emphasize the quantized nature of the problem and choose the (small) set $\mathbb{U}=\{-0.4,0,0.4\}$. This requires only 1 bit in each sampling interval corresponding to a 0.4 increment or decrement of the input signal since $0 \in \mathbb{U}$ can be specified by not sending anything. We also need to send address information for the tank whose input is to be changed, which requires $2 \mathrm{~b}$ for our application. Hence, the down-link data rate will be $1.5 \mathrm{~b} / \mathrm{s}$, i.e., $3 \mathrm{~b}$ every $2 \mathrm{~s}$.

We control the four tanks by means of the MPNC with horizon $\mathrm{N}_{\mathrm{P}}=2$. We remove from the possible control actions the possibility of sending no control action to any plant, i.e., we remove the zero value from $\mathbb{V}$. This provides the system with

${ }^{6}$ Note that this is deliberately specified as a very poorly quantized system to highlight performance issues. 

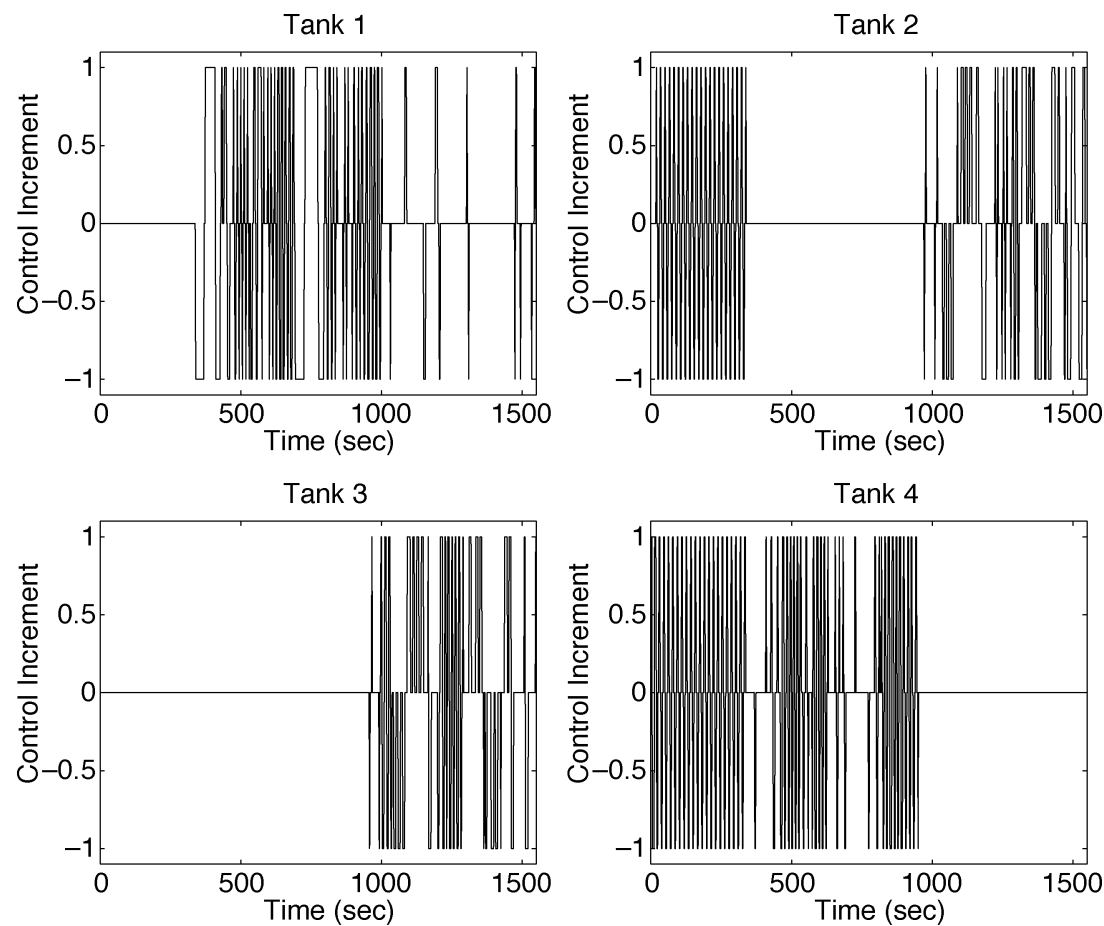

Fig. 9. Control increments.
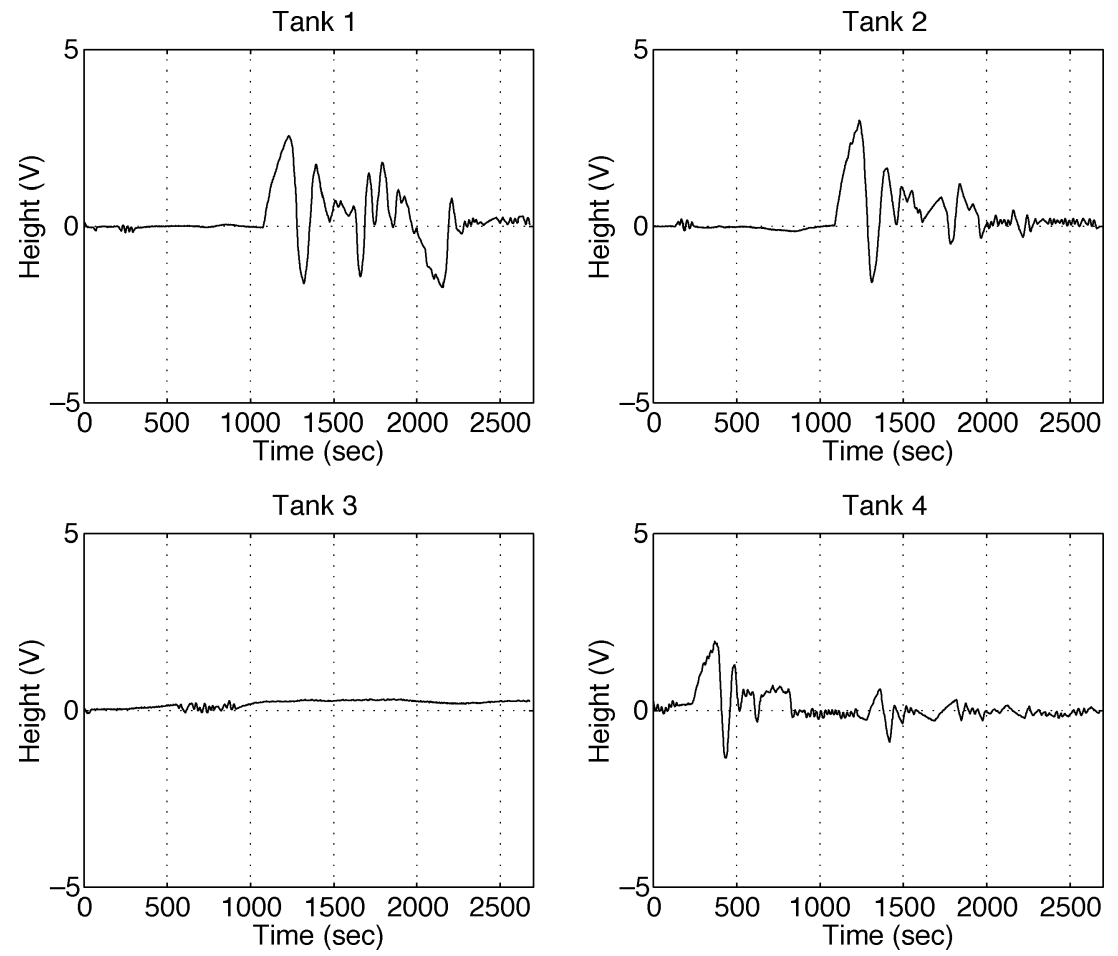

Fig. 10. Actual levels.

some degree of excitation when the plant output is close to the setpoint, which is needed to improve state estimation performance.

Gaussian noise with a variance of 0.04 is deliberately added to the measured signal prior to quantization to provide a small dithering signal.

\section{A. Stochastic Estimator}

The stochastic estimator parameters are chosen as follows:

$$
x_{00}=\left[\begin{array}{l}
0 \\
0
\end{array}\right] \quad P_{00}=\left[\begin{array}{cc}
0.9 & 0 \\
0 & 1.5
\end{array}\right] \quad Q=\left[\begin{array}{cc}
10^{-8} & 0 \\
0 & 10^{-8}
\end{array}\right]
$$

$R=0.04$ [see (4)], $\mathrm{N}_{\mathrm{M}}=7$ (see Section II-A3b). 

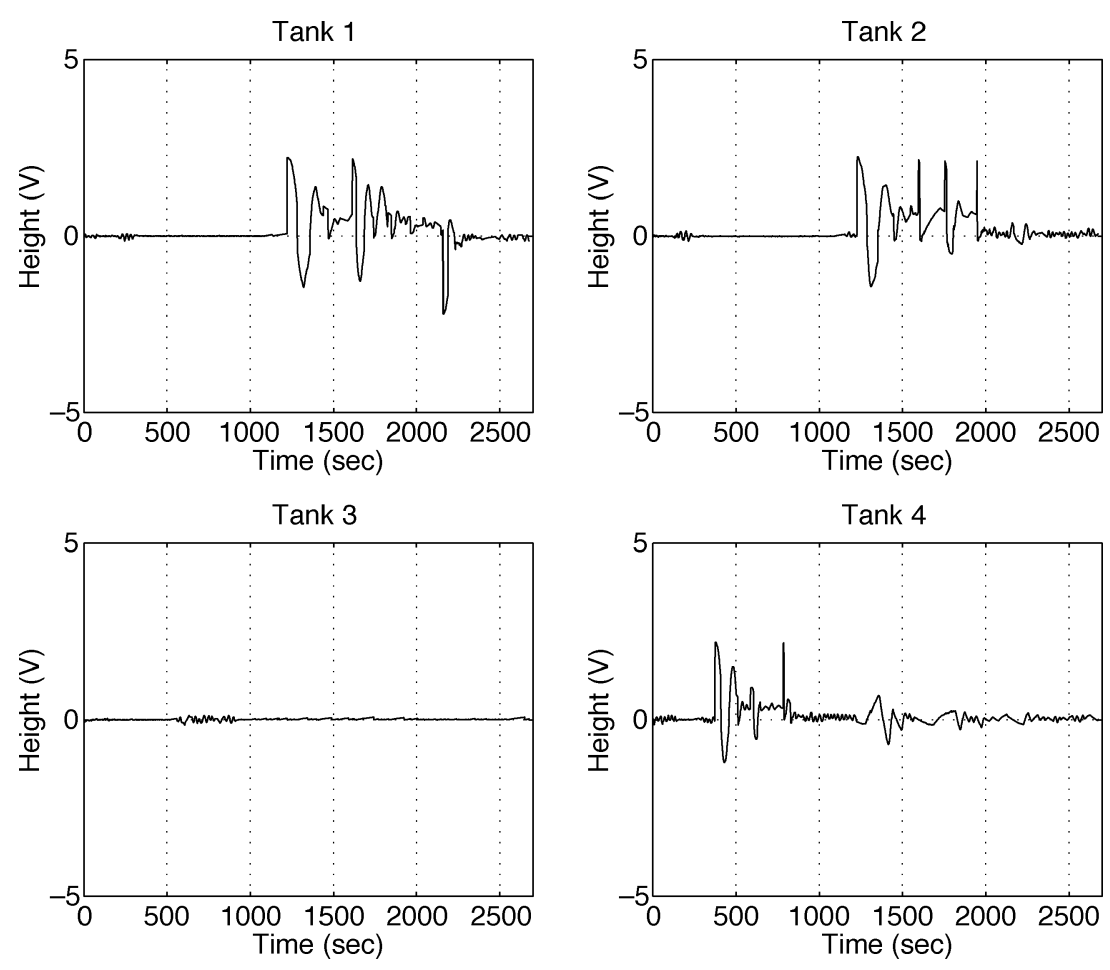

Fig. 11. Estimated levels.
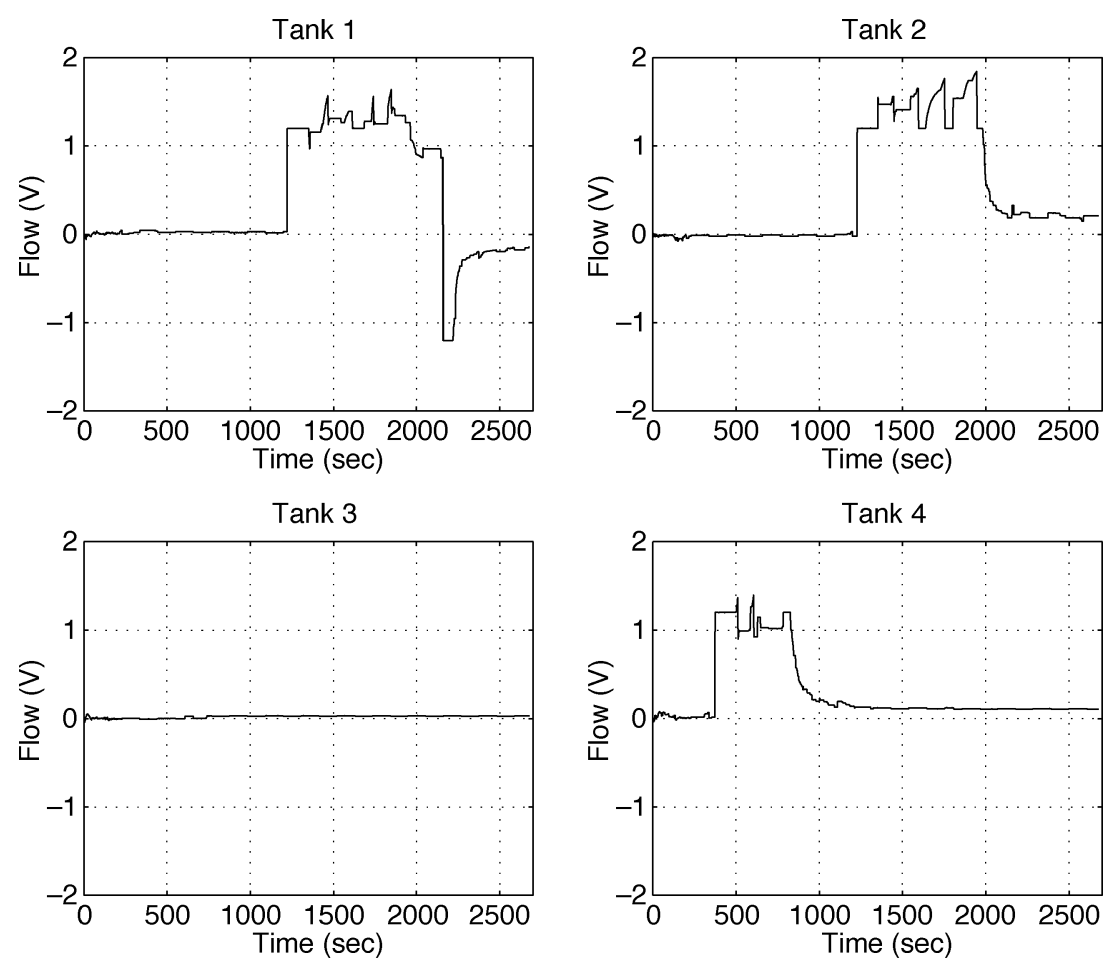

Fig. 12. Estimated disturbance inflow.

The number of samples drawn from the Gaussian pdf $p_{y_{k-\mathrm{N}_{\mathrm{M}}+1}, \ldots, y_{k}}\left(\gamma_{1}, \ldots, \gamma_{\mathrm{N}_{\mathrm{M}}}\right)$ at each discrete-time instant, $\mathrm{N}_{\mathrm{S}}$, is 20000 .

We simulated the system with the MC estimator as described in Section II-A. We found that the disturbance flow estimates appeared extremely noisy for the parameters used, to the point where the system performance became unacceptable. To smooth out the disturbance flow estimates, we included a low-pass filter with transfer function $G(\rho)=0.08 /\left(1-0.92 \rho^{-1}\right)$, and fed the output of this filter to the controller.

The simulation results are shown in Figs. 6 and 7. Initially, all disturbance flows are set to 0 . At 210 seconds, a disturbance flow of 1.5432 is applied to Tank 1 and is then reset to 0 at $710 \mathrm{~s}$. At $900 \mathrm{~s}$, two disturbance flows of 1.367 are simultaneously applied to Tanks 2 and 3 . They are both changed to -1.367 at $1146 \mathrm{~s}$ and back to 0 at $1500 \mathrm{~s}$. We discuss the results in Section IV-C, after 

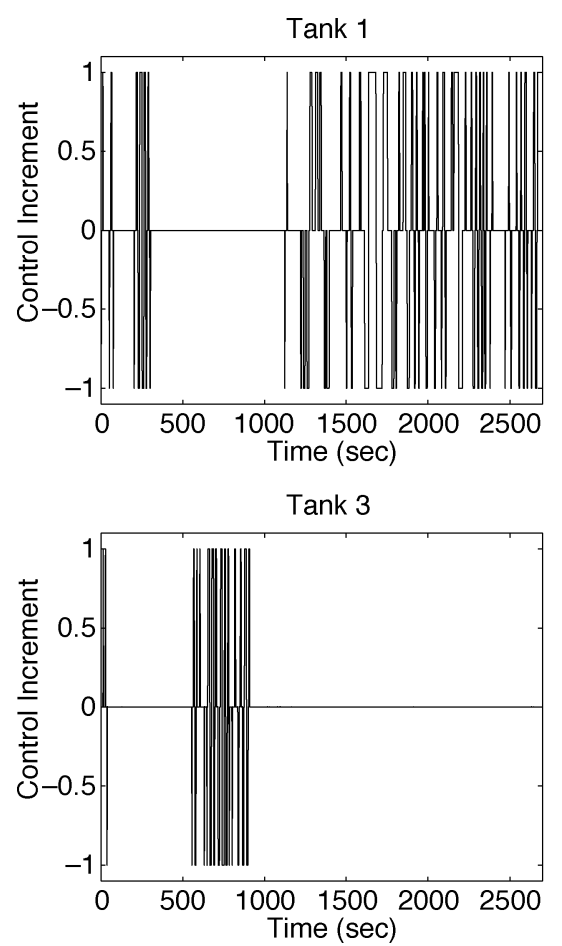

Fig. 13. Control increments sent over network.

we present the corresponding results obtained with the deterministic estimator.

\section{B. Deterministic Estimator}

We next use the deterministic estimator described in Section II-B to provide the state estimates.

To account for the dithering noise, a region expansion of $\epsilon=$ 0.6 is utilized in the estimator [see (27)]. We also assume the model accuracy to be sufficiently good to allow for $\delta=0$ [see (26)]. The observer length, $\mathrm{N}$, is set to 100 and we run 2 parallel observers $\left(\mathrm{N}_{\mathrm{O}}=2\right)$ to reduce computational complexity as in Section II-B3. The convex polytope $\mathcal{H}$ was defined as

$$
\mathcal{H}=\left\{\left[\begin{array}{l}
x^{1} \\
x^{2}
\end{array}\right] \in \mathbb{R}^{2}:\left[\begin{array}{c}
-5 \\
-2.5
\end{array}\right] \leq\left[\begin{array}{l}
x^{1} \\
x^{2}
\end{array}\right] \leq\left[\begin{array}{c}
5 \\
2.5
\end{array}\right]\right\}
$$

where -5 and 5 represent empty- and full-tank fluid levels, respectively, and -2.5 and 2.5 represent the minimum and maximum disturbance flow, respectively.

Since we seek to regulate the system to a point which lies on the border between quantization regions, we would expect to receive, on average, the same amount of measurements from each of these regions (because of the included dithering signal). If we keep receiving the same quantized measurement during an extended period of time, it is reasonable to assume that the system is not performing well. Whenever this situation occurs, we reset the $\mathrm{N}_{\mathrm{O}}$ observers and let the estimator start again from that point. The number of identical quantized measurements to receive before resetting is adopted as 62 . Note that this value is similar to the system's time constant.

The simulation results are shown in Figs. 8 and 9. We applied the same disturbance patterns as for the stochastic estimator (see Section IV-A).
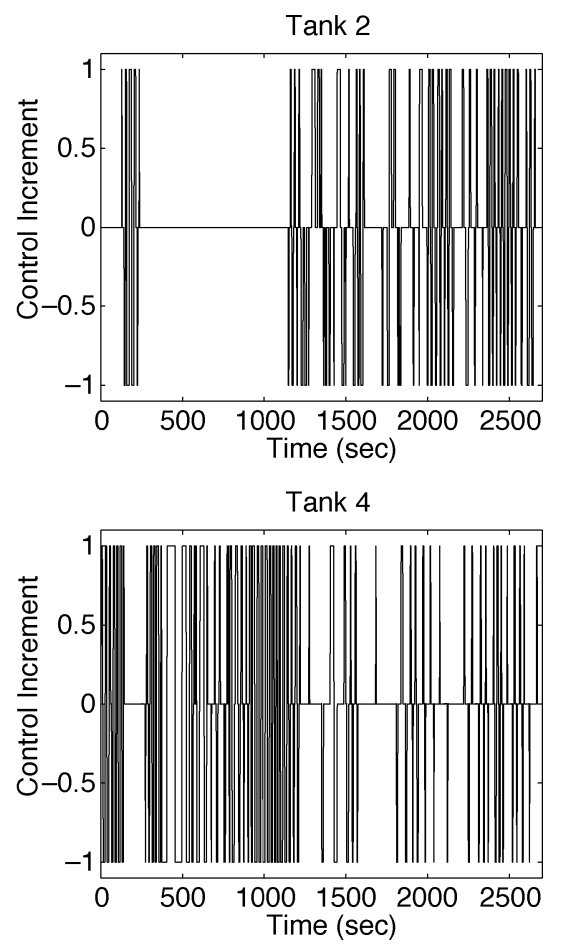

\section{Discussion}

Closed-loop performance is acceptable with either of the estimators. The stochastic estimator was more computationally demanding. Also, this estimator appears to detect the large steptype disturbances better but has worse steady-state characteristics as evidenced by the oscillating behavior in Fig. 6. Finally, the "attention" of the controller appears more focused in Fig. 9 for the deterministic estimator compared to Fig. 7 for the stochastic estimator.

\section{EXPERIMENTAL RESULTS}

In this section, we consider a practical experiment undertaken on a pilot-scale plant where level control for four similar tanks was configured. As with the computer simulations in Section IV, we study both the up-link and down-link problems of controlling the plants over a limited bandwidth channel. Because of the oscillating behavior of the stochastic estimator in our simulations, together with the higher computational effort needed (see Section IV-C), we implement only the deterministic estimator on the practical system.

The control system consists of a central controller connected to the plants by a digital network. Communications between the controller and plant is over the IEEE 802.3 standard Ethernet, 10 Base-T configuration utilizing the TCP/IP protocol. The communications interface employed was that of a client/server style architecture. The controller is the client and the plants are defined as servers. The control interface is provided by ProcessACT, which is a system for rapid prototyping of control system designs. ProcessACT also provides the network support here for the communications between the controller and plants. Thus, our NCS consisted of the controller, implemented in ProcessACT, running on a desktop PC with each plant also 

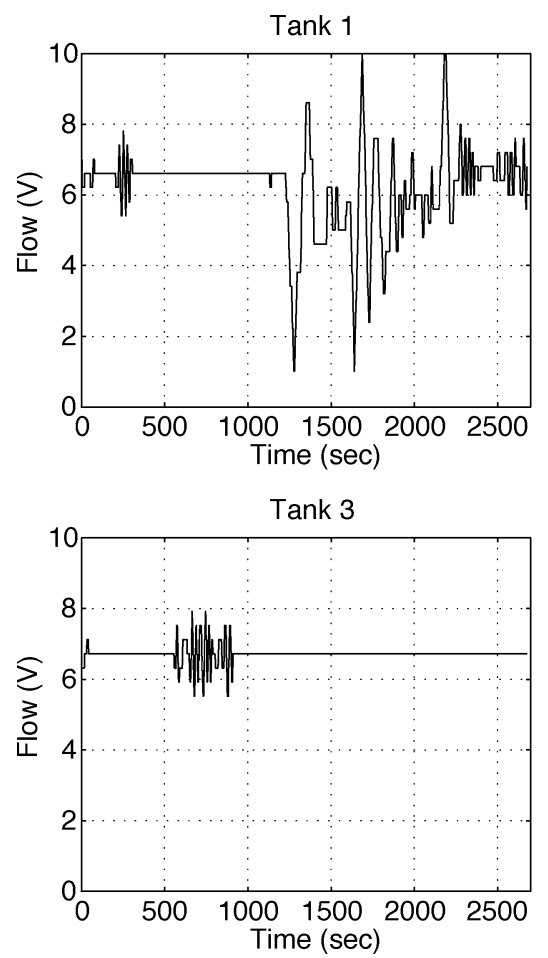

Fig. 14. Control signal at actuators.

having a dedicated PC to act as a communication gateway for the plant actuators.

To simulate the effect of a limited bandwidth network, we artificially reduced the Ethernet bandwidth by restricting the times at which data is sent and received. This sampling period was set to $2 \mathrm{~s}$. As in our simulations (Section IV), only coarsely quantized data is transmitted in either direction.

Once the tanks have reached the desired level $(0 \mathrm{~V})$ after the initial start-up, an unmeasured inflow disturbance was introduced into Tank 4 at approximately $250 \mathrm{~s}$ and removed at approximately $800 \mathrm{~s}$. The effect of this can be clearly seen in the actual tank level shown in Fig. 10 (Note that the data shown in Fig. 10 was not available to the control law but is shown here to illustrate the resulting performance). Fig. 11 shows the estimated tank levels (utilized by the controller). It can be seen that the observer detects the disturbance at approximately $370 \mathrm{~s}$. Fig. 12 shows the estimated disturbance. Once the disturbance is detected the controller begins to act. This can be observed in Fig. 13 which shows the control signal increments sent to each plant. Note that Tank 4 begins to receive attention once the disturbance is detected. The control signal applied to the actuators, i.e., after integration, is shown in Fig. 14.

Disturbances were next applied to Tanks 1 and 2 simultaneously at approximately $1080 \mathrm{~s}$ and removed at $1950 \mathrm{~s}$. The effect of these disturbances can be appreciated in the actual levels, as shown in Fig. 10. Again, the estimator can be seen (see Fig. 12) to lock onto the disturbances. The controller then pays attention to the tanks in most need, in this case Tanks 1 and 2 (see the control increments in Fig. 13).

We remark that in these practical experiments we did initially experience difficulties since the control signals at the controller did not match the reconstructed control signals at the actuators. This may have been caused by missing data in the down-link.
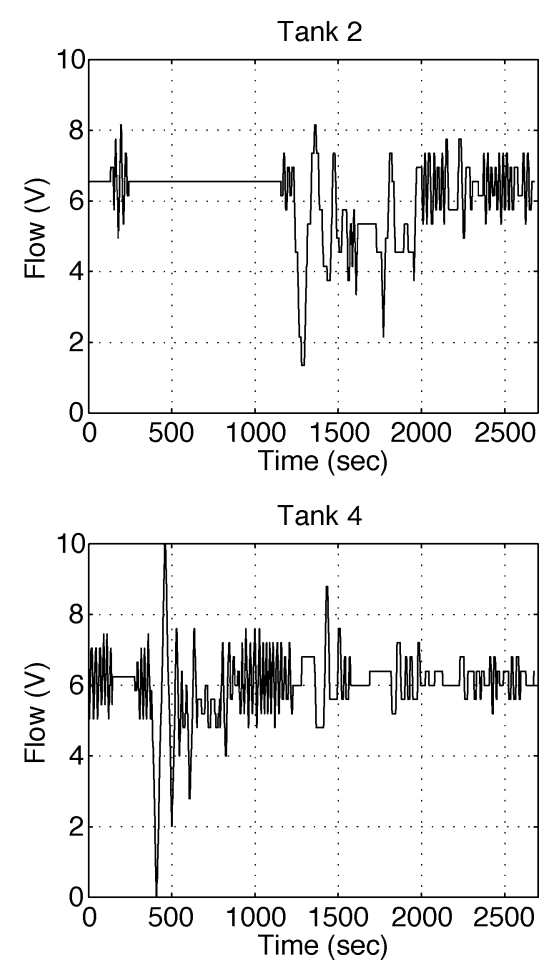

Thus, the actual plant inputs were provided as additional information to the controller as discussed in Remark 12.

\section{CONCLUSION}

This paper has described a moving horizon approach to networked control system design. Both up-link and down-link problems have been addressed. For the up-link problem, two alternative nonlinear state estimation methods have been developed in which only quantized measurements need to be transmitted. The choice between these alternatives is a function of prior knowledge about the nature of the noise and computational requirements. For the down-link, the control actions have been allocated by solving an optimization problem which effectively decides "which actuator should be addressed" and, for that actuator, "by how much (in quantized steps) the current control signal needs to be incremented." The algorithms have been tested on a laboratory-scale setup, which proved to be a challenging practical exercise. The experiments turned out to be informative in their own right.

\section{APPENDIX \\ PROOF OF THEOREM 2}

As in many stability results for controllers that operate in a receding horizon manner (see, e.g., [17]) our proof relies on showing that the sequence of optimal costs defined as

$$
\left(J_{\mathrm{N}_{\mathrm{P}}}^{\mathrm{opt}}\right)_{\ell} \triangleq J_{\mathrm{N}_{\mathrm{P}}}\left(\Delta \overrightarrow{\mathbf{u}}_{\ell \ell \ell+\mathrm{N}_{\mathrm{P}}-1}^{\mathrm{opt}}\right), \ell \in \mathbb{N}
$$

constitutes a Lyapunov function for the controlled noiseless plant model. 
At time $\ell$, and given $x_{\ell}$ and $u_{\ell-1}$, optimization yields the sequence

$$
\begin{aligned}
& \Delta \overrightarrow{\mathbf{u}}_{\ell: \ell+\mathrm{N}_{\mathrm{P}}-1}^{\mathrm{opt}} \\
& \quad=\left[\left(\Delta u_{\ell}\right)^{T}\left(\Delta u_{\ell+1}\right)^{T}, \ldots,\left(\Delta u_{\ell+\mathrm{N}_{\mathrm{P}}-1}\right)^{T}\right]^{T} \in \mathbb{V}^{\mathrm{N}_{\mathrm{P}}}
\end{aligned}
$$

and the cost $\left(J_{\mathrm{N}_{\mathrm{P}}}^{\mathrm{opt}}\right)_{\ell}$. Following the receding horizon principle, the first increment, $\Delta u_{\ell}$ is implemented, giving rise to $x_{\ell+1}=$ $A x_{\ell}+B u_{\ell}$ and $u_{\ell}=u_{\ell-1}+\Delta u_{\ell}$.

Next, at sample $\ell+1$, consider the related sequence

$$
\begin{aligned}
\Delta u^{\natural}=\left[\left(\Delta u_{\ell+1}\right)^{T}\left(\Delta u_{\ell+2}\right)^{T}\right. & \\
& \left.\ldots\left(\Delta u_{\ell+\mathrm{N}_{\mathrm{P}}-1}\right)^{T}\left(u^{\star}-u_{\ell+\mathrm{N}_{\mathrm{P}}-1}\right)^{T}\right]^{T}
\end{aligned}
$$

which belongs to $\mathbb{V}^{N_{P}}$, due to the constraint (58). With this choice, all predicted states $x_{\ell+i}^{\prime}, i=1, \ldots, \mathrm{N}_{\mathrm{P}}$ are equal in steps $\ell$ and $\ell+1$. The last value is given by

$$
\begin{aligned}
x_{\ell+\mathrm{N}_{\mathrm{P}}+1}^{\prime} & =A x_{\ell+\mathrm{N}_{\mathrm{P}}}^{\prime}+B\left(u_{\ell+\mathrm{N}_{\mathrm{P}}-1}+u^{\star}-u_{\ell+\mathrm{N}_{\mathrm{P}}-1}\right) \\
& =A x_{\ell+\mathrm{N}_{\mathrm{P}}}^{\prime}+B u^{\star}
\end{aligned}
$$

where we have utilized (42).

Thus, it holds that

$$
\begin{aligned}
J_{\mathrm{N}_{\mathrm{P}}}\left(\Delta u^{\natural}\right)= & \left(J_{\mathrm{N}_{\mathrm{P}}}^{\mathrm{opt}}\right)_{\ell}+\left\|x_{\ell+\mathrm{N}_{\mathrm{P}}+1}^{\prime}-x^{\star}\right\|_{P}^{2}-\left\|x_{\ell+\mathrm{N}_{\mathrm{P}}}^{\prime}-x^{\star}\right\|_{P}^{2} \\
& +\left\|y_{\ell+\mathrm{N}_{\mathrm{P}}+1}^{\prime}-r^{\star}\right\|^{2}-\left\|y_{\ell+1}-r^{\star}\right\|^{2} \\
& +\left\|u_{\ell+\mathrm{N}_{\mathrm{P}}}-u^{\star}\right\|_{R}^{2}-\left\|u_{\ell}-u^{\star}\right\|_{R}^{2} \\
= & \left(J_{\mathrm{N}_{\mathrm{P}}}^{\mathrm{opt}}\right)_{\ell}+\left\|A x_{\ell+\mathrm{N}_{\mathrm{P}}}^{\prime}+B u^{\star}-x^{\star}\right\|_{P+C^{T} C}^{2} \\
& -\left\|x_{\ell+\mathrm{N}_{\mathrm{P}}}^{\prime}-x^{\star}\right\|_{P}^{2} \\
& -\left(\left\|x_{\ell+1}-x^{\star}\right\|_{C^{T} C}^{2}+\left\|u_{\ell}-u^{\star}\right\|_{R}^{2}\right)
\end{aligned}
$$

after substituting (55) and (60).

On the other hand, (54) yields the relation $A x_{\ell+\mathrm{N}_{\mathrm{P}}}^{\prime}+B u^{\star}-$ $x^{\star}=A x_{\ell+\mathrm{N}_{\mathrm{P}}}^{\prime}-A x^{\star}$. Hence

$$
\begin{aligned}
J_{\mathrm{N}_{\mathrm{P}}}\left(\Delta u^{\natural}\right)= & \left(J_{\mathrm{N}_{\mathrm{P}}}^{\mathrm{opt}}\right)_{\ell}+\left\|x_{\ell+\mathrm{N}_{\mathrm{P}}}^{\prime}-x^{\star}\right\|_{A^{T} P A+A^{T} C^{T} C A-P}^{2} \\
& -\left(\left\|x_{\ell+1}-x^{\star}\right\|_{C^{T} C}^{2}+\left\|u_{\ell}-u^{\star}\right\|_{R}^{2}\right) \\
= & \left(J_{\mathrm{N}_{\mathrm{P}}}^{\mathrm{opt}}\right)_{\ell}-\left(\left\|x_{\ell+1}-x^{\star}\right\|_{C^{T} C}^{2}+\left\|u_{\ell}-u^{\star}\right\|_{R}^{2}\right)
\end{aligned}
$$

using (59). that

Due to optimality, it follows that $\left(J_{\mathrm{N}_{\mathrm{P}}}^{\mathrm{opt}}\right)_{\ell+1} \leq J_{\mathrm{N}_{\mathrm{P}}}\left(\Delta u^{\natural}\right)$, so

$$
\begin{aligned}
\left(J_{\mathrm{N}_{\mathrm{P}}}^{\mathrm{opt}}\right)_{\ell+1} & -\left(J_{\mathrm{N}_{\mathrm{P}}}^{\mathrm{opt}}\right)_{\ell} \\
\leq & -\left(\left\|x_{\ell+1}-x^{\star}\right\|_{C^{T} C}^{2}+\left\|u_{\ell}-u^{\star}\right\|_{R}^{2}\right) \leq 0 .
\end{aligned}
$$

Hence, $\lim _{\ell \rightarrow \infty}\left(J_{\mathrm{N}_{\mathrm{P}}}^{\mathrm{opt}}\right)_{\ell}$ exists and $\left(J_{\mathrm{N}_{\mathrm{P}}}^{\mathrm{opt}}\right)_{\ell+1}-\left(J_{\mathrm{NP}_{\mathrm{P}}}^{\mathrm{opt}}\right)_{\ell} \rightarrow 0$. As a consequence of (61), both $\left\|x_{\ell+1}-x^{\star}\right\|_{C^{T} C}^{2}$ and $\left\|u_{\ell}-u^{\star}\right\|_{R}^{2}$ converge to 0 , which proves the theorem.

\section{REFERENCES}

[1] "Special section on networks and control," IEEE Control Syst. Mag., vol. 21, pp. 57-99, Feb. 2001.

[2] H. Ishii and B. A. Francis, Limited Data Rate in Control Systems With Networks. New York: Springer-Verlag, 2002.

[3] R. M. Gray and D. L. Neuhoff, "Quantization," IEEE Trans. Inform. Theory, vol. 44, pp. 2325-2383, Oct. 1998.

[4] R. E. Curry, Estimation and Control With Quantized Measurements. Cambridge, MA: M.I.T. Press, 1970.
[5] D. F. Delchamps, "Extracting state information from a quantized output record," Syst. Control Lett., vol. 13, pp. 365-372, 1989.

[6] _- "Stabilizing a linear system with quantized state feedback," IEEE Trans. Automat. Contr., vol. 35, pp. 916-924, July 1990.

[7] R. Koplon and E. D. Sontag, "Linear systems with sign-observations," SIAM J. Control Optim., vol. 31, no. 5, pp. 1245-1266, 1993.

[8] O. Feely, "A tutorial introduction to nonlinear dynamics and chaos and their application to sigma-delta modulators," Int. J. Circuit Theory Applicat., vol. 25, pp. 347-367, 1997.

[9] J. Baillieul, "Feedback coding for information-based control: operating near the data-rate limit," in Proc. IEEE Conf. Decision Control, 2002, pp. 3229-3236.

[10] L. W. Liou and A. Ray, "A stochastic regulator for integrated communication and control systems," Trans. ASME, vol. 113, pp. 604-619, 1991.

[11] J. Nilsson, B. Bernhardsson, and B. Wittenmark, "Stochastic analysis and control of real-time systems with random time delays," Automatica, vol. 34 , no. 1 , pp. $57-64,1998$.

[12] H. Chan and Ü. Özgüner, "Closed-loop control of systems over a communications network with queues," Int. J. Control, vol. 62, no. 3, pp. 493-510, 1995.

[13] G. C. Goodwin and K. S. Sin, Adaptive Filtering Prediction and Control. Upper Saddle River, NJ: Prentice-Hall, 1984.

[14] K. J. Åström, "Riemann and Lebesgue sampling," presented at the Advances in system theory: A symposium in honor of Sanjoy K. Mitter, Cambridge, MA, Oct. 15-16, 1999.

[15] J. A. De Doná, G. C. Goodwin, and M. M. Serón, Constrained Control \& Estimation-An Optimization Perspective. New York: SpringerVerlag, 2004.

[16] J. M. Maciejowski, Predictive Control With Constraints. Upper Saddle River, NJ: Prentice-Hall, 2002.

[17] D. Q. Mayne, J. B. Rawlings, C. V. Rao, and P. O. M. Scokaert, "Constrained model predictive control: optimality and stability," Automatica, vol. 36, no. 6, pp. 789-814, 2000.

[18] C. V. Rao, J. B. Rawlings, and D. Q. Mayne, "Constrained state estimation for nonlinear discrete-time systems- stability and moving horizon approximations," IEEE Trans. Automat. Contr., vol. 48, pp. 246-258, Feb. 2003.

[19] G. Ferrari-Trecate, D. Mignone, and M. Morari, "Moving horizon estimation for hybrid systems," IEEE Trans. Automat. Contr., vol. 47, pp. 1663-1676, Oct. 2002.

[20] W. K. Hastings, "Monte Carlo sampling methods using Markov chains and their applications," Biometrika, vol. 57, no. 1, pp. 97-109, 1970.

[21] F. C. Schweppe, "Recursive state estimation: unknown but bounded errors and system inputs," IEEE Trans. Automat. Contr., vol. AC-13, pp. 22-28, Jan. 1968.

[22] D. P. Bertsekas and I. B. Rhodes, "Recursive state estimation for a setmembership description of uncertainty," IEEE Trans. Automat. Contr., vol. AC-16, pp. 117-128, Feb. 1971.

[23] M. Milanese and A. Vicino, "Optimal estimation theory for dynamic systems with set membership uncertainty: an overview," Automatica, vol. 27, no. 6, pp. 997-1009, 1991.

[24] J. P. Norton, Ed., International Journal Adaptive Control Signal Processing Special Issue: Bounded-Error Estimation: Issue 1. New York: Wiley, 1994, vol. 8.

[25] E. Walter and H. Piet-Lahanier, "Guaranteed linear and nonlinear parameter estimation from bounded error data: a survey," in Proc. IEEE Int. Symp. Circuits, Systems, 1993, pp. 774-777.

[26] R. W. Brockett, "Stabilization of motor networks," in Proc. IEEE Conf. Decision Control, 1995, pp. 1484-1488.

[27] D. Hristu and K. Morgansen, "Limited communication control," Syst. Control Lett., vol. 37, no. 4, pp. 193-205, 1999.

[28] D. E. Quevedo, G. C. Goodwin, and J. A. De Doná, "Finite constraint set receding horizon control," Int. J. Robust Nonlinear Control, vol. 14, no. 4, pp. 355-377, Mar. 2004.

[29] H. Haimovich, G. C. Goodwin, and D. E. Quevedo, "Moving horizon Monte Carlo state estimation for linear systems with output quantization," in Proc. IEEE Conf. Decision Control, 2003, pp. 4859-4864.

[30] D. E. Quevedo, G. C. Goodwin, and J. S. Welsh, "Minimizing down-link traffic in networked control systems via optimal control techniques," in Proc. IEEE Conf. Decision Control, 2003, pp. 1200-1205.

[31] H. Haimovich, G. C. Goodwin, and J. S. Welsh, "Set-valued observers for constrained state estimation of discrete-time systems with quantized measurements," in Proc. Asian Control Conf., 2004, pp. 1947-1955. 
[32] B. D. O. Anderson and J. Moore, Optimal Filtering. Upper Saddle River, NJ: Prentice-Hall, 1979.

[33] A. H. Jazwinski, Stochastic Processes and Filtering Theory. New York: Academic, 1970.

[34] J. A. De Doná, G. C. Goodwin, and A. Jutan, "A rolling horizon state estimator with constraint horizon one," in Proc. 15th IFAC World Congr., 2002.

[35] G. C. Goodwin and J. C. Agüero, "State and parameter estimation for linear and nonlinear systems," in Int. Conf. Control, Automation, Robotics, and Vision, ICARCV, 2002, pp. B-1-B-12.

[36] J. G. Proakis, Digital Communications. New York: McGraw-Hill, 1995.

[37] R. M. Gray, "Dithered quantizers," IEEE Trans. Inform. Theory, vol. 39, pp. 805-812, Mar. 1993.

[38] E. Fogel and Y. F. Huang, "On the value of information in system identification—bounded noise case," Automatica, vol. 18, no. 2, pp. 229-238, 1982

[39] A. V. Savkin and I. R. Petersen, "Set-valued state estimation via a limited capacity communication channel," IEEE Trans. Automat. Contr., vol. 48, pp. 676-680, Apr. 2003.

[40] H. Piet-Lahanier and E. Walter, "Exact description of feasible parameter sets and minimax estimation," Int. J. Adapt. Control Signal Processing, vol. 8, pp. 5-14, 1994.

[41] B. Grünbaum, Convex Polytopes. New York: Wiley, 1967.

[42] S. Boyd and L. Vandenberghe, Convex Optimization. New York: Springer-Verlag, 2004.

[43] L. Palopoli, A. Bicchi, and A. Sangiovanni-Vincentelli, "Numerically efficient control of systems with communication constraints," in Proc. IEEE Conf. Decision Control, 2002, pp. 1626-1631.

[44] D. E. Quevedo, G. C. Goodwin, and J. S. Welsh, "Design issues in a networked control system architecture," in Proc. IEEE Conf. Control Applications, 2004, to be published.

[45] A. Gersho and R. M. Gray, Vector Quantization and Signal Compression. Norwell, MA: Kluwer, 1992.

[46] A. Bicchi, A. Marigo, and B. Piccoli, "On the reachability of quantized control systems," IEEE Trans. Automat. Contr., vol. 47, pp. 546-562, Apr. 2002.

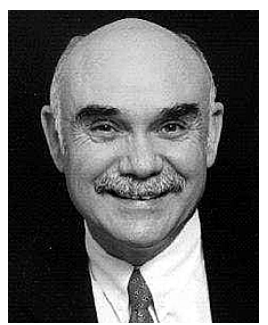

Graham C. Goodwin (M'74-SM'84-F'86) received the B.Sc. degree in physics, the B.E. degree in electrical engineering, and the Ph.D. degree from the University of New South Wales, New South Wales, Australia, in 1965, 1967, and 1971, respectively.

From 1970 to 1974 , he was a Lecturer in the Department of Computing and Control, Imperial College, London, U.K. Since 1974, he has been with the Department of Electrical and Computer Engineering, The University of Newcastle, Newcastle, NSW, Australia. He is the coauthor of seven monographs, four edited volumes, and several hundred technical papers. He is currently Professor of Electrical Engineering and Associate Director of the Centre for Integrated Dynamics and Control at the University of Newcastle.

Dr. Goodwin was the recipient of several international prizes including the USA Control Systems Society 1999 Hendrik Bode Lecture Prize, a Best Paper award by the IEEE TRANSACTIONS ON AUTOMATIC CONTROL, and a Best Paper Award by the Asian Journal of Control. He was also the recipient of an ARC Federation Fellowship, and he is an Honorary Fellow of the Institute of Engineers, Australia, a Fellow of the Australian Academy of Science, a Fellow of the Australian Academy of Technology, Science, and Engineering, a Member of the International Statistical Institute, and a Fellow of the Royal Society, London.

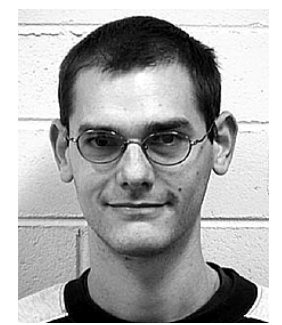

Hernan Haimovich (S'98-M'01) was born in Rosario, Argentina, in 1977. He received the Ingeniero Electrónico (electronics engineering) degree from the Universidad Nacional de Rosario in 2001, achieving the top first grade-point average in his graduation year. He is currently working toward the $\mathrm{Ph} . \mathrm{D}$. degree with the School of Electrical Engineering and Computer Science, The University of Newcastle, Callaghan, NSW, Australia.

During his undergraduate studies, he participated in a country-wide science and technology competition and was awarded with a special distinction. He worked as a Teaching Assistant at the Universidad Nacional de Rosario from 1999 to 2002. His research interests include quantized control systems, differential flatness, and nonlinear control.

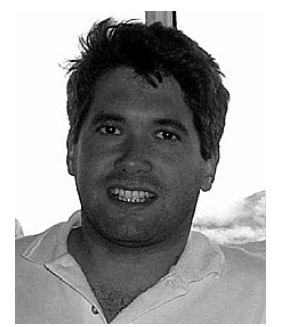

Daniel E. Quevedo (S'02) received the Ingeniero Civil Electrónico and Magister en Ingeniería Electrónica degrees from the Universidad Técnica Federico Santa María, Valparaíso, Chile, in 2000. During his time at the university, he was supported by a full scholarship from the alumni association and, upon graduating, received several university-wide prizes. $\mathrm{He}$ is currently working toward the $\mathrm{Ph} . \mathrm{D}$. degree with the School of Electrical Engineering and Computer Science, The University of Newcastle, Callaghan, NSW, Australia.

$\mathrm{He}$ has lectured at both of the aforementioned universities in several courses related to automatic control. He has also worked experience at the VEW Energie AG, Dortmund, Germany, and the Cerro Tololo Inter-American Observatory, Chile. His main research interests cover several areas of automatic control, signal processing and communications.

Mr. Quevedo received the IEEE Conference on Decision and Control Best Student Paper Award in 2003, and was also a finalist for the same award in 2002. In 2003, he was awarded the University of Newcastle Faculty of Engineering and Built Environment Prize for the Best International Conference Paper.

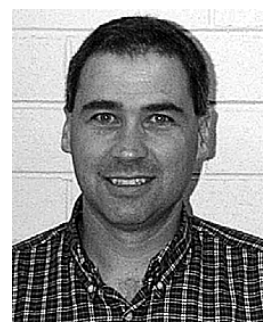

James S. Welsh (M'02) was born in Maitland, Australia, in 1965. He received the B.E. degree (Hons. I) in electrical engineering from The University of Newcastle, Callaghan, NSW, Australia, in 1997, and has recently submitted his Ph.D. dissertation for examination in electrical engineering at same university.

He gained industrial experience from 1981 to 1997 with Bloomfield Collieries, AES, and TYP Pty, Ltd. Australia. During the last two years, he has been actively involved in a research project on Powertrain Control with the Centre for Integrated Dynamics and Control, the Universty of Newcastle. His research interests include auto-tuning, closed-loop identification, and process control. He has recently been appointed as a Lecturer in the School of Electrical Engineering and Computer Science, The University of Newcastle.

Mr. Welsh was awarded the University of Newcastle Faculty of Engineering and Built Environment Prize for the Best International Journal Paper in 2003. In 1997, he won the Institute of Engineers Australia National Undergraduate Thesis Prize in Automation, Control, and Instrumentation. 